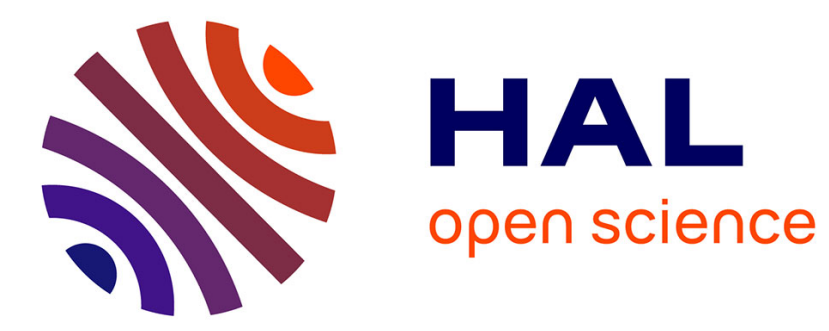

\title{
Numerical Study of Hypersonic Boundary-Layer Transition Delay through Second-Mode Absorption
}

Romain Fiévet, Hughes Deniau, Jean-Philippe Brazier, Estelle Piot

\section{To cite this version:}

Romain Fiévet, Hughes Deniau, Jean-Philippe Brazier, Estelle Piot. Numerical Study of Hypersonic Boundary-Layer Transition Delay through Second-Mode Absorption. AIAA SCITECH 2020, Jan 2020, ORLANDO, United States. 10.2514/6.2020-2061 . hal-02486673

\section{HAL Id: hal-02486673 https://hal.science/hal-02486673}

Submitted on 21 Feb 2020

HAL is a multi-disciplinary open access archive for the deposit and dissemination of scientific research documents, whether they are published or not. The documents may come from teaching and research institutions in France or abroad, or from public or private research centers.
L'archive ouverte pluridisciplinaire HAL, est destinée au dépôt et à la diffusion de documents scientifiques de niveau recherche, publiés ou non, émanant des établissements d'enseignement et de recherche français ou étrangers, des laboratoires publics ou privés. 


\title{
Numerical study of hypersonic boundary-layer transition delay through second-mode absorption
}

\author{
Romain Fiévet*1, Hughes Deniau ${ }^{1}$, Jean-Philippe Brazier ${ }^{1}$, Estelle Piot ${ }^{1}$ \\ ${ }^{1}$ The French Aerospace Laboratory (ONERA/DMPE) - Université de Toulouse, F-31055, Toulouse, France
}

\begin{abstract}
Numerical time-domain impedance boundary conditions are developed within a highorder spectral difference flow solver. They are designed to accurately replicate the acoustic response of ultrasonically-absorbent coatings over a broad spectral range. The coupling of this class of high-fidelity wall boundary conditions with high-order numerical methods has enabled their use in aero-acoustics applications. This solver is used to perform direct numerical simulations (DNS) of a hypersonic boundary layer and analyze the stabilizing effects of acoustically absorbent materials on the laminar-to-turbulent transition. The damping effectiveness of such complex porous coating on key frequencies of interest is investigated, as well as its effect on the mean flow. It is found that the second-mode instability, which dominates the high-Mach number flow regime, is strongly suppressed at the expense of higher far-field noise radiation. The solutions obtained by means of DNS are also found to compare favorably with linearized stability theory.
\end{abstract}

\section{Introduction}

One of the primary concerns when designing hypersonic vehicles is the strong aero-thermal load suffered during atmospheric flights. The surface drag and heating requires the use of thermal protection system which constraints the vehicle weights, reducing its maximal payload and flight performance. Mitigating these fluid-structure exchange processes is, therefore, a key design consideration. Being diffusive by nature, they are known to be mitigated when the boundary layer remains laminar as opposed to being turbulent. ${ }^{1-3}$ Hence, the laminar-to-turbulent transition of hypersonic boundary layers (HBL) has been the focus of a large body of studies which has shed light on the underlying physics.

In absence of bypass forcing, as is common assumption for high-altitude high-speed flights, transition is caused by the spatial growth of unstable waves. ${ }^{4}$ At supersonic speeds, linear stability compressible theory was used by Mack ${ }^{5}$ to predict the existence of an infinite number of modes, amongst which some are unstable. The first unstabe mode can be considered as the hypersonic equivalent of Tollmien-Schlichting waves and is predominant below Mach 4. The second (and higher) unstable modes are acoustic in nature (i.e. compressible and inviscid) and dominate the transition process beyond Mach 4 . The latter modes occur when instabilities' phase speeds become supersonic relative to the flow velocity at the critical layer, defined as the location where the local flow velocity equals the sound speed. Such instabilities have been experimentally $3,6,7$ and numerically ${ }^{2,8-11}$ visualized as waves trapped between the wall and sonic line, producing rope-like structures around the critical layer. In fact, the wall-normal gradient of the acoustic impedance forms an impedance well ${ }^{12}$ inside the boundary layer which physically acts as an acoustic wave-guide. The resonating acoustic modes trapped inside it grow as they propagate and energy is progressively transferred from the mean laminar kinetic energy into acoustic energy until turbulence breaks down. ${ }^{12}$ Interestingly, wall cooling further destabilizes the higher modes contrary to the first viscous mode ${ }^{5,13}$ as it exacerbates the impedance well effect. As the second mode has a higher amplitude than the higher modes ${ }^{5}$ it is of critical interest to design second-mode canceling systems to delay the laminar-to-turbulent transition in HBL.

One of the most promising second-mode canceling mechanism is the use of porous coatings. The pores act as acoustic resonators and can be designed to filter out specific frequencies. It was theorized by Fedorov

\footnotetext{
*Postdoctoral Research Fellow, romain.fievet@onera.fr

Copyright (C) 2019 by Romain Fiévet. Published by the American Institute of Aeronautics and Astronautics, Inc. with permission.
} 
in $2001^{14}$ and experimentally validated by Rasheed et al. the following year ${ }^{15}$ in a Mach 5 conical flow configuration. This has encouraged further study and development of Ultrasonically Absorptive Coating (UAC) designed to absorb second-mode waves in order to delay transition in HBL. ${ }^{16-20}$ Importantly, it was observed that the wall porosity has a braodband effect on the acoustic field and coincidentally trigger first-mode ${ }^{8,16}$ and higher-modes ${ }^{21}$ instabilities. Hence, the coating's acoustic properties must be carefully optimized to respectively maximize/minimize the spectral canceling/reinforcement regimes.

Computational studies have also been able to capture the UAC stabilizing effect by either directly resolving the porous coating ${ }^{21,22}$ or by modeling its impedance as a boundary condition. ${ }^{23-27}$ The direct numerical simulation (DNS) of Brès et al. ${ }^{21}$ resolved the UAC damping effects using the former method by meshing the pores as uniformly-spaced cavities. This provided important insight on pore-to-pore interactions and revealed the presence of a slip-velocity induced instability in the high-porosity (pores hole to surface ratio) shallow-pores limit. However, the computational cost limited the scale of the simulation over a single second-mode wavelength, focusing on temporal rather than the more relevant spatial instabilities. To circumvent the need to mesh the porosity, time-domain impedance boundary conditions (TDIBC) mimicking the UAC response over a delimited spectral range have been successfully used in linear ${ }^{23,24,27}$ and nonlinear solvers alike. $^{25,26}$ Of particular interest is the recent work of Sousa et al. ${ }^{25,26}$ which performed a DNS aimed at capturing the whole transition region with TDIBC calibrated using experimental measurement of UAC impedance. Notably, their TDIBC was derived from the scattering operator rather than the impedance operator as was done in previous studies. ${ }^{23,24,27}$ The use of the scattering operator was proven by Monteghetti et al. ${ }^{28}$ to offer better numerical stability than the unbounded impedance operator in grazing flows. The multi-pole approach Sousa et al. used allows for a broadband resolution of the UAC spectral response, although no detail on the reflectivity and phase shift resolution was given. The impedance model did not account for thermoviscous effect inside the UAC either. Finally, in all these numerical studies, the TDIBC implementation relied on Euler theory: the treatment of the wall-normal diffusive flux and resulting spurious reflections at low reflectivity isothermal conditions was not addressed.

With this background, the current project aims at developing a state-of-the-art numerical platform to study boundary layer stability in hypersonic configurations and guide the design of optimal UAC, able to achieve flow's laminarity over the vehicle.

To this end, TDIBC derived from a strong formalism of the Navier-Stokes equations are implemented in a high-order discontinuous spectral difference (SD) solver. The SD method is highly-scalable and minimallydissipative, both of which are necessary conditions to run large-scale DNS and resolve the hydro-acoustic interactions within the transition region. The method also retains spectral-like accuracy ${ }^{29}$ on unstructured grids which allows to simulate complex geometries with ease. All these properties designate it as an ideal candidate to construct this numerical platform around. The implementation of the TDIBC follows the work of Monteghetti et al. ${ }^{30}$ and later development for the Navier-Stokes by Fiévet. ${ }^{31}$ They are designed to: 1) provide a broadband representation of the UAC spectral response over a range of interest (RoI), 2) use an impedance model accounting for frequency-dependent thermoviscous losses, 3) offer a clear discretization routine of the scattering operator generating a reasonable amount of hyper-parameters, 4) be properly coupled with the Navier-Stokes equations and 5) be efficiently implemented for massively parallel calculations.

Further, high-fidelity aero-acoustic numerical simulations require non-reflecting boundary conditions to avoid spurious reflections to contaminate the inner solution. This could feedback on the physical growth of unstable modes and artificially trigger instabilities. Typically, such boundary conditions are enforced through the celebrated family of characteristic boundary conditions for the Navier-Stokes equations (NSCBC). ${ }^{32}$ NSCBC were recently enabled with the SD method by Fiévet et al., ${ }^{31}$ and are used in this study for the fist time to study the laminar-to-turbulent transition.

Once developed, the solver is used to study the damping of second-mode instabilities in a Mach 5.5 hypersonic boundary layer by means of DNS. The configuration is chosen to mimick the experiment of Wagner et al. ${ }^{18}$ on a two-dimensional (2D) dihedral. It represents a $27 \mathrm{~km}$-altitude Mach 7.4 flight. Their UAC, which acoustic properties were measured in Sousa et al. ${ }^{25}$ is used to accurately calibrate the TDIBC over a broad spectral range. Its stabilizing properties are assessed as well as its coincident effects on the mean flow. Finally, the results obtained from the DNS are compared with the linear stability theory.

Importantly, this study is also meant to serve as a milestone before inverse-problem solving methods, under ongoing development, be coupled with the solver and used to design optimal UAC for target flight conditions.

The paper is organized as follows. First, a brief overview of numerical methods employed in the solver (SD 
and NSCBC) are presented. Second, the development of the TDIBC is thoroughly described. A third section presents the hypersonic cone configuration and mean flow results. The fourth and last section presents the results of the stability analysis.

\section{Numerical methods}

The high-order spectral difference code used in this project, JAGUAR (project of an Aerodynamic solver using $G$ eneral $U$ nstructured grids $A$ nd high orde $R$ schemes), is actively co-developed by ONERA and CERFACS (European Center of Research and Formation in Advanced Scientific Calculus) with the help of IMFT (Institute of Fluid Mechanics in Toulouse). Ongoing developments include combustion modules, real gas effects, further maturation of characteristic boundary conditions treatment, embedded automatic differentiation and adjoint methods (Cardesa and Airiau ${ }^{33}$ ). Benchmarking and validation test cases are available in the literature ${ }^{34,35}$. The SD method is presented first for the sake of completeness. Then, the governing equations for characteristic boundary conditions with the Navier-Stokes equations as implemented in JAGUAR are presented.

\section{A. Spectral Difference method}

Discontinuous finite element methods (DFEM) have gained much traction in the past years thanks to their ability to achieve locally arbitrarily-high orders of accuracy on unstructured grid. Such accuracy was previously only attainable with large-stencil finite difference (FD) schemes naturally restricted to structured grids, which prevented the use of complex geometries with high-order schemes.

The SD method resolves the strong form of the Navier-Stokes equations inside each mesh element, as in FD. The global algorithm of the $p$-order SD method is presented in Fig. 1 for an element in a one-dimensional (1D) mesh and briefly explained below.

During initialization, each element is transformed into a standardized $[0,1]$ domain. Then, $p+1$ solution and $p+2$ flux points (respectively called SP and FP) are distributed inside the element using the GaussLegendre quadrature points. Note that FP are placed at the element boundaries at 0 and +1 . The SP store the conservative variables which are marched in time while the FP serve as a base to reconstruct a continuous flux. However, the method does not assume that the conservative variables form a continuous solution across the computational domain.

At the start of a time sub-iteration, the conservative variables solution $U$ stored in the $p+1 \mathrm{SP}$ are used to reconstruct a $p$-order Lagrange polynom which represents the continuous solution across the element (globally discontinuous). It is then used to evaluate a continuous flux $F(U)$ at the $p+2$ FP. A Riemann problem is resolved at the element boundaries which provides element-to-element communication and ensures flux continuity across the whole computational domain. A $(p+1)$-order flux polynom is reconstructed from the flux values at the FP. The flux polynom is then derived at the solution point to march the solution in time.
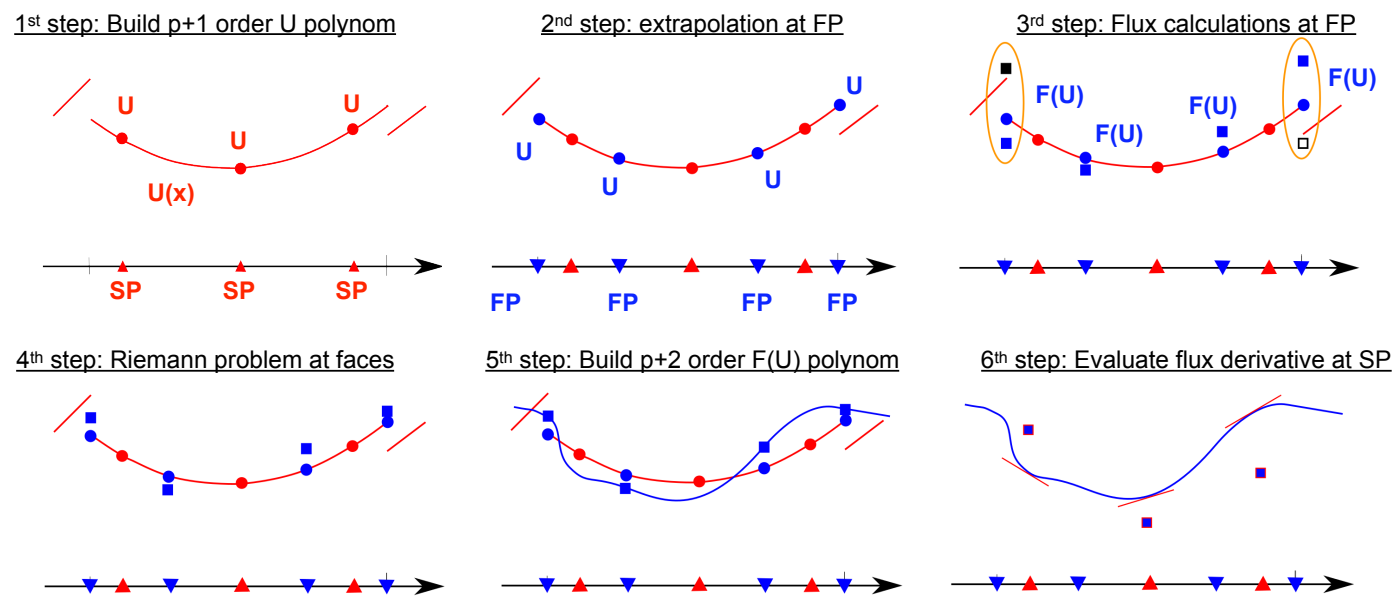

Figure 1. Global algorithm of the $\mathrm{p}=2 \mathrm{SD}$ method in $1 \mathrm{D}$ reproduced from Cassagne et al. ${ }^{34}$ 
Vanharen et al. ${ }^{29}$ have assessed the accuracy of the SD method for various polynom orders and compared it with spectral-like resolution $6^{\text {th }}$-order compact FD schemes. ${ }^{36,37}$ They showed that the $6^{\text {th }}$-order SD method is at least as accurate, albeit a local method suited for unstructured grids contrary to these FD methods. A spectral-like resolution implies that the numerical scheme causes minimal dissipation and dispersion errors. This is particularly important when resolving problems where a large range of wavenumbers are physically relevant to the problem, as in laminar-to-turbulent transition and aeroacoustics. Finally, a 6 -stage explicit Runge-Kutta scheme is used to march the solution in time.

\section{B. Characteristic Boundary Conditions for Navier-Stokes equations}

As explained in Sec. I, the use of non-reflecting numerical boundary conditions is of paramount importance in laminar-to-turbulent transition studies. In Fiévet et al., ${ }^{31}$ NSCBC were successfully used with the SD method: the reader is referred to their study for a thorough description of their implementation accompanied by validation test cases. A brief overview is offered below to introduce useful notations for Sec. III.

Specifically, the implementation of the NSCBC in JAGUAR follows the methodology of Kim and Lee ${ }^{38}$ which apply characteristic boundary conditions through the modification of flux derivatives at the boundaries. While originally intended for FD methods, their method is well suited for SD as well since characteristics can be evaluated with great accuracy at the boundary flux points using the local high-order extrapolation scheme.

The non-reacting Navier-Stokes equations are written in three-dimensional (3D) generalized coordinates $(\xi, \eta, \zeta)$ as:

$$
\begin{aligned}
\frac{\partial U}{\partial t} & +\left(\xi_{x} \frac{\partial E_{c}}{\partial \xi}+\xi_{y} \frac{\partial F_{c}}{\partial \xi}+\xi_{z} \frac{\partial G_{c}}{\partial \xi}\right)+\left(\xi_{x} \frac{\partial E_{d}}{\partial \xi}+\xi_{y} \frac{\partial F_{d}}{\partial \xi}+\xi_{z} \frac{\partial G_{d}}{\partial \xi}\right) \\
& +\left(\eta_{x} \frac{\partial E_{c}}{\partial \eta}+\eta_{y} \frac{\partial F_{c}}{\partial \eta}+\eta_{z} \frac{\partial G_{c}}{\partial \eta}\right)+\left(\eta_{x} \frac{\partial E_{d}}{\partial \eta}+\eta_{y} \frac{\partial F_{d}}{\partial \eta}+\eta_{z} \frac{\partial G_{d}}{\partial \eta}\right) \\
& +\left(\zeta_{x} \frac{\partial E_{c}}{\partial \zeta}+\zeta_{y} \frac{\partial F_{c}}{\partial \zeta}+\zeta_{z} \frac{\partial G_{c}}{\partial \zeta}\right)+\left(\zeta_{x} \frac{\partial E_{d}}{\partial \zeta}+\zeta_{y} \frac{\partial F_{d}}{\partial \zeta}+\zeta_{z} \frac{\partial G_{d}}{\partial \zeta}\right)=0
\end{aligned}
$$

where $\left(E_{c}, F_{c}, G_{c}\right)$ and $\left(E_{d}, F_{d}, G_{d}\right)$ respectively correspond to the convective and diffusive fluxes in the physical coordinate system $(x, y, z)$. Without any loss of generality, when applying NSCBC at a $\xi$-normal face, the characteristic decomposition of the $\xi$-normal convective flux is expressed as:

$$
\left(\xi_{x} \frac{\partial E_{c}}{\partial \xi}+\xi_{y} \frac{\partial F_{c}}{\partial \xi}+\xi_{z} \frac{\partial E_{c}}{\partial \xi}\right)=P_{U} \Delta P_{U}^{-1} \frac{\partial U}{\partial \xi},
$$

with $\Delta$ being the diagonalized matrix of $\frac{\partial E_{c}}{\partial U}$ 's eigenvalues and $P_{U}$ the associated eigenvectors forming the transformation matrix. The vector of characteristic $\mathcal{L}$ is defined as:

$$
\mathcal{L}=\Delta P_{U}{ }^{-1} \frac{\partial U}{\partial \xi} .
$$

Likewise, the transformation matrix is used to define the following quantities:

$$
\begin{aligned}
& W=P_{U}{ }^{-1} U, \\
& \mathcal{T}_{c}=P_{U}{ }^{-1}\left[\left(\eta_{x} \frac{\partial E_{c}}{\partial \eta}+\eta_{y} \frac{\partial F_{c}}{\partial \eta}+\eta_{z} \frac{\partial G_{c}}{\partial \eta}\right)+\left(\zeta_{x} \frac{\partial E_{c}}{\partial \zeta}+\zeta_{y} \frac{\partial F_{c}}{\partial \zeta}+\zeta_{z} \frac{\partial G_{c}}{\partial \zeta}\right)\right], \\
& \mathcal{T}_{d}=P_{U}{ }^{-1}\left[\left(\eta_{x} \frac{\partial E_{d}}{\partial \eta}+\eta_{y} \frac{\partial F_{d}}{\partial \eta}+\eta_{z} \frac{\partial G_{d}}{\partial \eta}\right)+\left(\zeta_{x} \frac{\partial E_{d}}{\partial \zeta}+\zeta_{y} \frac{\partial F_{d}}{\partial \zeta}+\zeta_{z} \frac{\partial G_{d}}{\partial \zeta}\right)\right], \\
& \mathcal{D}=P_{U}^{-1}\left(\xi_{x} \frac{\partial E_{d}}{\partial \xi}+\xi_{y} \frac{\partial F_{d}}{\partial \xi}+\xi_{z} \frac{\partial G_{d}}{\partial \xi}\right) .
\end{aligned}
$$

Hence, Eq. 1 can be rewritten in characteristic space as:

$$
\frac{\partial W}{\partial t}+\mathcal{L}+\mathcal{T}_{c}+\mathcal{T}_{d}+\mathcal{D}=0
$$


The convective and diffusive tangent fluxes $\mathcal{T}_{c}$ and $\mathcal{T}_{d}$ are evaluated from the inner solution, while the normal diffusive fluxes $\mathcal{D}$ usually rely on Dirichlet or Neumann conditions to evaluate the momentum and thermal energy gradients. Applying NSCBC consists in resolving systems of $\mathcal{L}$ at the boundary FP verifying the desired physical condition. For instance, pure non-reflecting outflow conditions imply no entering wave, i.e. all entering characteristics set to zero.

These equations are used to derive characteristic boundary conditions using the following steps (a $\xi$ normal boundary is considered to illustrate their method). First, Eq. 3 is used to provide an initial guess for the $\mathcal{L}$ using flux derivatives extrapolated from the inner solution. Second, the $\mathcal{L}$ are modified using classical NSCBC conditions. ${ }^{32}$ The new $\xi$-normal convective characteristics are labeled $\mathcal{L}^{*}$. Third, Eq. 3 is used again to obtain the corresponding corrected $\xi$-normal convective flux derivative $\frac{\partial E_{c}^{*}}{\partial \xi}$. Fourth, this quantity is returned into Eq. 1 to march the solution in time.

\section{Implementation of time-domain impedance boundary conditions}

The implementation of TDIBC with the SD scheme follows the works of Monteghetti et al. ${ }^{30}$ and later Fiévet et al. ${ }^{31}$ A summary of both works is presented below for the sake of completeness.

As explained my Monteghetti et al., ${ }^{30}$ a TDIBC is comprised of three components. First, a physical impedance model has to be chosen to describe the UAC in Laplace space. Second, the said model is discretized and converted into the time-domain. This usually amounts to evaluate a convolution product in time. Last, a semi-discrete formulation of the TDIBC is needed to couple it with the numerical flow solver.

Some useful notations and basic concepts pertaining to acoustics for linearized Euler equations are given first before describing each aforementioned step.

\section{A. Acoustic impedance conditions for the linearized Euler equations}

In acoustics, the impedance $Z$ of a medium characterizes its reaction to a traveling acoustic pressure perturbation. For a mono-dimensional (1D) medium where the impedance depends only on the perturbation's angular frequency $\omega$ and space, it can be defined as:

$$
z(x, j \omega)=\frac{p^{\prime}(x, j \omega)}{u^{\prime}(x, j \omega)}
$$

where $p^{\prime}$ and $u^{\prime}$ are the Fourier-transformed acoustic pressure and velocity perturbations in a unperturbed flow at Mach $M_{0}$. Further, the acoustic pressure perturbation can be decomposed into a sum of right-running and left-running waves:

$$
p^{\prime}(t)=p^{\prime} e^{j \omega t}=p^{+} e^{j \omega t-k_{1+M_{0}} x}+p^{-} e^{j \omega t+k_{1-M_{0}} x}
$$

with wavenumber $k_{1+M_{0}}=\omega / c_{0} /\left(1+M_{0}\right)$ and $k_{1-M_{0}}=\omega / c_{0} /\left(1-M_{0}\right)$. The reflection coefficient $\beta$ is defined as the ratio between the amplitudes of the reflected and incident waves, it can also be derived from the impedance as:

$$
\beta[z]=\frac{z-\rho_{0} c_{0}}{z+\rho_{0} c_{0}}
$$

where $\rho_{0}$ and $c_{0}$ are the medium's reference density and speed of sound, respectively.

Finally, the impedance and scattering operator $\mathcal{Z}$ and $\mathcal{B}$ respectively define the following positive-real functions in the time-domain:

$$
p^{\prime}(t)=\left[z \star u^{\prime}\right](t)=\mathcal{Z}\left[u^{\prime}\right](t)
$$

and

$$
v^{+}(t)=\left[\beta \star v^{-}\right](t)=\mathcal{B}\left[v^{-}\right](t)
$$

where $v^{+}=p^{\prime}-\rho_{0} c_{0} u^{\prime}$ and $v^{-}=p^{\prime}+\rho_{0} c_{0} u^{\prime}$ are the reflected and incident wave amplitudes, respectively. They correspond to the characteristics of the linearized Euler equations (LEE) traveling at the speed of sound in opposite directions. Both $\mathcal{Z}$ and $\mathcal{B}$ define a TDIBC. 
As pointed out by Monteghetti et al. ${ }^{30}$ it is more appropriate to adopt a $\mathcal{B}$-formalism rather than a $\mathcal{Z}$-formalism when constructing a TDIBC due to $\beta$ being bounded contrary to $z$. This proved to relax the restriction on the Courant Friedrichs Lewy (CFL) number ${ }^{30}$ which is of paramount importance to CFD applications.

\section{B. Impedance modeling of an UAC}

The first component of a TDIBC consists of its impedance model, which relates the wall pressure fluctuations with the wall normal velocity. In the present study, the UAC is modeled as a set of narrow cavities which form a honeycomb core. Each cavity behaves as an acoustic wave-guide with a rigid end which effectively damps its resonant frequencies as in Jones et al. ${ }^{39}$ The honeycomb's cells have a thickness $l_{c}$, diameter $d_{c}$ and porosity $\tau_{c}$. The porosity measures the ratio between the perforated and whole surface. The impedance of the liner reads:

$$
z_{\mathrm{SDOF}}=\frac{z_{c}}{\sigma_{c}} \operatorname{coth}\left(\mathrm{j} k_{c} l_{c}\right)
$$

with the following fractional wavenumber $k_{c}(s)$ ( $s=j \omega$ referring to the Laplace variable herein):

$$
j k_{c}(s) l_{c}=a_{0}+a_{\frac{1}{2}} \sqrt{s}+a_{1} s .
$$

where $a_{0}$ models frequency-independent losses, $a_{1 / 2}$ corresponds to frequency-dependent losses from thermoviscous dissipation and diffraction, and $a_{1}$ characterizes the system's mass reactance and does not induce any energy loss. The three coefficients can be estimated with different theoretical and empirical models.

\section{Delayed multi-pole representation of an impedance model}

The second step of the TDIBC construction consists in discretizing the reflection coefficient $\beta[z(s)]$ in the time-domain to derive the scattering operator $\mathcal{B}$. To this end, $\beta$ is first decomposed into an infinite sum of complex poles using an oscillatory-diffusive representation (ODR) as defined by Monteghetti et al. ${ }^{28,30}$ For the sake of conciseness, the reader is referred to these papers to obtain a detailed derivation of the ODR. The ODR of the reflection coefficients reads as:

$$
\beta(s)=\beta_{\infty}+\sum_{n \in I} \frac{r_{1, n}}{s-s_{n}}+\int_{0}^{\infty} \frac{\mu_{1}(\xi)}{s+\xi} \mathrm{d} \xi+e^{-s \tau}\left(\sum_{n \in I} \frac{r_{2, n}}{s-s_{n}}+\int_{0}^{\infty} \frac{\mu_{2}(\xi)}{s+\xi} \mathrm{d} \xi\right) .
$$

where the complex oscillatory poles $s_{n}$ are the zeros of $z+\rho_{0} c_{0}$ and the diffusive poles $\xi$ are arbitrarily chosen to span the spectral range of interest for a given configuration. The delay $\tau$ corresponds to the back-and-forth wave traveling time inside the cavity. Finally, the weights $r_{n}$ and $\mu_{n}$ are evaluated to fit the analytical function (Eq. 8).

It is important to note that no approximation has been made yet: the model is exactly represented by an infinite sum of oscillatory and diffusive poles. The oscillatory poles form conjugate pairs which guarantees the bounded-reality of the associated scattering operator. Further, an approximated reflection coefficient $\tilde{\beta}$ is naturally derived by only using a finite number of $N_{\xi}$ diffusive and $N_{s}$ oscillatory poles $\tilde{\xi}_{k}$ and $\tilde{s}_{n}$ :

$$
\tilde{\beta}(s)=\tilde{\beta}_{\infty}+\sum_{n=1}^{N_{s}} \frac{\tilde{r}_{1, n}}{s-\tilde{s}_{n}}+\sum_{k=1}^{N_{\xi}} \frac{\tilde{\mu}_{1, k}}{s+\tilde{\xi}_{k}}+e^{-s \tilde{\tau}}\left(\sum_{n=1}^{N_{s}} \frac{\tilde{r}_{2, n}}{s-\tilde{s}_{n}}+\sum_{k=1}^{N_{\xi}} \frac{\tilde{\mu}_{2, k}}{s+\tilde{\xi}_{k}}\right)
$$

Importantly, the ODR discretization offers the possibility to optimize the poles and weights values to match an experimental dataset. An example of the effectiveness of such data-driven discretization is provided in Fig.2. As previously mentioned, the test case considered in this paper and presented in Sec. IV is based on the experimental work of Wagner et al. ${ }^{18}$ The spectral properties of the UAC they use are quantified in, ${ }^{25}$ and are now used to calibrated the TDIBC's poles and weights. The ODR of $\tilde{\beta}$ shown in Fig.2 was performed using 4 diffusive poles $\xi$ and 8 oscillatory pairs of poles. This corresponds to a 20-scalar model, including the poles weights and excluding the complex conjugates, which captures the complex broadband response of the UAC with great accuracy up to $1 \mathrm{MHz}$. All these scalars are given in the appendix in Tab. 2. Using half the number of poles would result in a discretization similarly accurate up to $500 \mathrm{kHz}$.

The underlying interest of the ODR of $\beta$ lies in the transformation of the convolution product (Eq. 10) into a sum of auxiliary functions solutions of ordinary differential equations (ODE) as demonstrated 

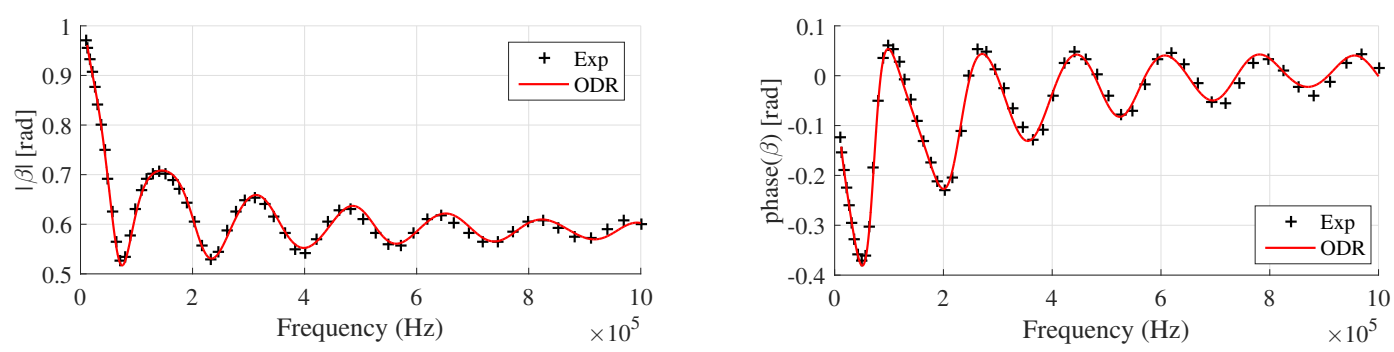

Figure 2. Discrete ODR of the UAC from Sousa et al. ${ }^{25}$ using 4 diffusive and 8 oscillatory poles.

by Monteghetti et al. ${ }^{28}$ This powerful result permits to approximate the scattering operator $\tilde{\mathcal{B}}$ (i.e. the TDIBC) with ODE using the same explicit time-marching scheme as the flow conservative variables $U$. The evaluation of $\tilde{\mathcal{B}}$ based on $\tilde{\beta}$ reads as:

$$
\tilde{\mathcal{B}}(t)=\tilde{\beta}_{\infty} v^{-}(t)+\sum_{n=1}^{N_{s}} \tilde{r}_{1, n} \phi\left(t,-\tilde{s}_{n}\right)+\sum_{k=1}^{N_{\xi}} \tilde{\mu}_{1, k} \phi\left(t, \tilde{\xi}_{k}\right)+\sum_{n \in I} \tilde{r}_{2, n} \phi\left(t-\tilde{\tau},-\tilde{s}_{n}\right)+\sum_{k=1}^{N_{\xi}} \tilde{\mu}_{2, k} \phi\left(t-\tilde{\tau}, \tilde{\xi}_{k}\right)
$$

where the $\phi$ are evaluated using:

$$
\left\{\begin{array}{l}
\partial_{t} \phi=-\zeta \phi(t, s)+v^{-}(t) \quad(t>0, \zeta \in \mathbb{C}) \\
\phi(t, 0)=\phi(0, s)=0
\end{array}\right.
$$

for any pole $\zeta$. The delay is resolved by transporting at the medium's speed of sound $c_{0}$ the $\phi$ variables over another spatial coordinate $\theta$ of length equal to $\tau / c_{0}$. To this end, another set of auxiliary functions $\psi$ is introduced:

$$
\psi\left(t, \zeta, l_{\tilde{\tau}}\right)=\phi(t-\tilde{\tau}, \zeta) .
$$

The additional auxiliary functions $\psi$ are solutions of the following transport equation:

$$
\left\{\begin{array}{l}
\partial_{t} \psi(t, \zeta, \theta)=c_{0} \partial_{\theta} \psi(t, \zeta, \theta) \quad\left(t>0, \theta \in\left[0, l_{\tilde{\tau}}\right], \zeta \in \mathbb{C}\right) \\
\psi(t, \zeta, 0)=\phi(t, \zeta) \\
\psi(0, \zeta, \theta)=0
\end{array}\right.
$$

This amounts to resolving an advection problem for each pole at each FP located on a TDIBC face. The discretization scheme of the $\theta$-space determines the delay and maximum frequency that can be resolved through Eqs. 18. Its length $l_{\tilde{\tau}}$ verifies $l_{\tilde{\tau}}=\tilde{\tau} c_{0}$ which determines the cavity depth for a given delay and speed of sound. The number of elements per wavelength (EPW), $N_{e}$, determines the cut-off frequency $f_{\max }=\left(N_{e} \times \mathrm{EPW}\right) /(2 \tilde{\tau})$ with a minimum of EPW $=2$ needed to avoid aliasing. The numerical flux is evaluated using a $6^{t h}$-order SD scheme with upwinding. Since there is no guarantee that the ODR yields a passive TDIBC outside the $\xi$-bounds, it is suggested to choose $f_{\max } \leq \max (|\xi|)$ to naturally filter out higher frequencies. A performance study was carried by Fiévet et al. ${ }^{31}$ who found that EPW $=8$ resulted in dissipative losses on the order $0.01 \%$ of the incidence wave's amplitude for a delay equal to 10 periods. They found that the computational cost of the advection problems was greatly mitigated by the use of array-wide operations on contiguous data, clever CPU-partitioning and use of the same time-marching scheme as the main simulation.

Alternatively, Douasbin et al. ${ }^{40}$ showed that the delay operator can be approximated by a multi-pole function, in similar fashion as $\beta$ 's ODR. Their approach is more efficient memory-wise than the direct resolution presented above which requires to store information at all the $\theta$-elements' solution points. On the other hand, the direct approach uses only one real hyper-parameter $\tau$ while the multi-pole approach uses an unknown number of complex poles and weights determined by an iterative process. The pole's numbers, values and corresponding weights are adjusted until the target delay is sufficiently well resolved. The use of complex poles also causes instabilities outside the poles bounds and/or when an insufficient number of poles 
is used. Importantly, the direct approach permits to resolve all delays up to $\tilde{\tau}=l_{\tilde{\tau}} / c_{0}$ by simply extracting $\psi$ at a shorter $\theta$, and resolving a higher delay simply requires to add $\theta$-elements in order to increase $l_{\tilde{\tau}}$. This allows for a real-time feedback which will prove necessary for future adjoint-based optimization applications, hence this method was chosen.

To conclude, the work presented in this section permits to recast the scattering operator into a numericallyapproximated discrete form embedded within the time-marching scheme of a CFD solver.

\section{Coupling of the TDIBC with the Navier-Stokes equations}

The third and last TDIBC component is the coupling with the flow solver, i.e. the evaluation of the conservative variables numerical flux $F(U)$ at the TDIBC faces. To this end a semi-discrete formulation was derived $^{31}$ to be used with the Navier-Stokes equations. For the sake of simplicity, the method is presented for a $1 \mathrm{D}$ case where $W^{+}$and $W^{-}$respectively define the Navier-Stokes $(u+c)$-traveling incident and the $(u-c)$-traveling reflected characteristics. $c$ is the flow speed of sound and way vary in space time. They read as:

$$
\left\{\begin{array}{l}
\partial W^{+}=\partial p+\rho c \partial u \\
\partial W^{-}=\partial p-\rho c \partial u
\end{array}\right.
$$

The previous steps detailed in Sec. B and C permit to evaluate in time and space the scattering operator $\tilde{\mathcal{B}}$ valid for the linearized Euler equations. As $\tilde{\mathcal{B}}$ is a linear function of $v^{-}$, it follows naturally that:

$$
\partial_{t} W^{+}=\mathcal{B}\left(\partial_{t} W^{-}\right)
$$

which is reorganized using Eq. 5 into:

$$
[\mathcal{L}+\mathcal{D}]^{+}=\mathcal{B}\left(\left[\mathcal{L}+\mathcal{D}+\mathcal{T}_{c}+\mathcal{T}_{d}\right]^{-}\right)-\left[\mathcal{T}_{c}+\mathcal{T}_{d}\right]^{-}
$$

The scattering operator is then comprised of a weighted sum of auxiliary functions $\phi$ and $\psi$ evaluated with Eqs. 18 and 16 where $v^{-}$has been replaced by $W^{-}$. This coupling method injects the TDIBC by adjusting the numerical flux at the wall using Eq. 5. In this sense, the TDIBC is embedded within the NSCBC and can be considered as a subclass.

\section{E. Test cases}

The robustness of the NSCBC-embedded and ODR-based TDIBC was extensively verified. ${ }^{31}$ Yet, in the context of this study, the robustness of the TDIBC specifically derived from Fig. 2 (and Tab. 2) is investigated. To this end, an impedance tube test case is run. It consists of a single-period right-running harmonic wave of frequency $f$ convected onto the TDIBC. The other end of the domain is treated as a non-reflecting pressure outflow. The computational domain is discretized with 50 cells per wavelength and four frequencies $f=[25,50,100,200] \mathrm{kHz}$ are investigated.

The reflected wave profiles are sampled and compared with the analytical solution derived from resolving the monodimensional Riemann problem of the linearized Euler equations as in Monteghetti. ${ }^{28}$ The comparisons between these profiles are presented in Fig. 3 for all four frequencies considered and serve as a testament to the TDIBC robustness used herein.

\section{Stability analysis of a hypersonic boundary layer}

The high-fidelity numerical methods presented above, integrated within the same flow solver, are now used to simulate a HBL and study the UAC stabilization properties. To this end, an unperturbed hypersonic boundary-layer flow field is first developed. Then, linear stability theory (LST) is used to predict the range of frequency of interests corresponding to the most unstable modes along the streamwise direction. Last, the HBL is excited by the means of a wall blowing mechanism in order to trigger harmonic instabilities and study their interaction with a UAC simulated by a TDIBC. 

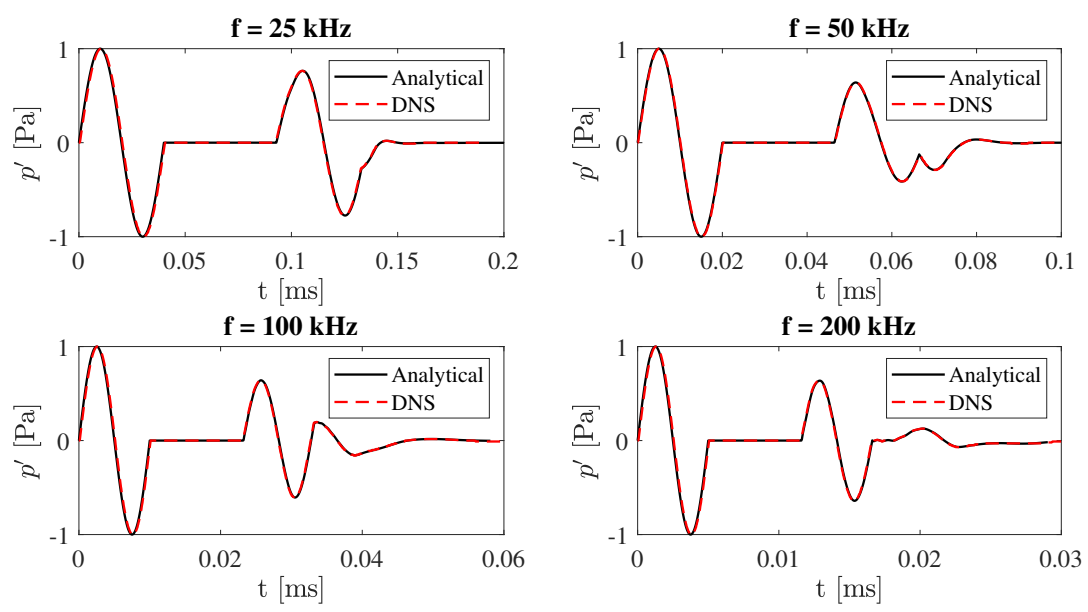

Figure 3. Comparison between analytical and DNS pressure perturbations signals from an impedance tube numerical experiment ran at different frequencies.

The TDIBC constructed in Sec. III was defined by a series of poles, weights, reflectivity and delay calibrated to match the broadband properties of the carbon fiber porous material developped at the German Aerospace Laboratory. ${ }^{18,19}$ This UAC was successfully used to delay the laminar-to-turbulent transition on a conical surface in an experiment at the High-Enthalpy Goettingen facility mimicking a high altitude Mach 7.4 flight. Specifically, the similarly designed numerical experiment of Sousa et al. ${ }^{26}$ replicated 28 km-altitude Mach 7.4 flight conditions. The numerical experiment considered herein is designed to match as closely as possible the conditions of these aforementioned studies.

\section{A. Description of the numerical experiment protocol}

The far-field conditions of all numerical simulations presented from this point on are those given in Tab. 1. The model's geometry is chosen to be a $7^{\circ}$ half-angle $120 \mathrm{~cm}$-long dihedral. While the second-mode properties are known to differ from a cone to dihedral model, this allows to decrease the simulation's computational cost by adopting a 2D Cartesian mesh instead of a 3D cylindrical mesh. As the objectives of this seminal study are not to perform a direct comparison with experimental data, this is deemed acceptable.

\begin{tabular}{|c|c|c|c|c|}
\hline \hline$R e_{x}\left[\mathrm{~m}^{-1}\right]$ & $M_{\infty}$ & $p_{\infty}[\mathrm{Pa}]$ & $T_{\infty}[\mathrm{K}]$ & $u_{\infty}[\mathrm{m} / \mathrm{s}]$ \\
\hline $4.06 \times 10^{6}$ & 7.4 & 2129 & 268 & 2422 \\
\hline \hline
\end{tabular}

Table 1. Far-field conditions for the hypersonic dihedral simulation.

A schematic of the numerical configurations used throughout this study is presented in Fig. 4. Its protocol comprises the following orderly steps:

1. A precursor solution of the dihedral flow is run with the high-enthalpy solver $\operatorname{CEDRE}^{41,42}$ until time and grid convergence are reached. It uses a second-order spatial method and a second-order implicit time scheme. Its purpose is to serve as the unperturbed reference flows and initial state to subsequent runs. The wall isothermal temperature $T_{w}=300 \mathrm{~K}$ for the remainder of the study.

2. The wall-normal profiles are extracted along the wall. A ONERA in-house linear stability theory (LST) solver, MAMOUT, ${ }^{43}$ is then used to determine the frequency range of interest to be investigated with the DNS, alongside the corresponding second-mode growth region.

3. The precursor's solution within the aforementioned region is extracted and used to define the DNS' boundary conditions and initial state. A first unperturbed DNS is then run to develop a time-converged, unperturbed HBL with JAGUAR. Importantly, it uses characteristic boundary conditions all along the isothermal no-slip wall wall: that is the TDIBC uses a fully-reflective $(\mathcal{B}=1)$ hard wall condition.

4. The UAC is then activated, and its impact on the mean field is quantified. In absence of any timeinstability, this case is expected to yield a solution identical to that obtained with the hard wall 
boundary conditions. This is a direct consequence of the TDIBC's well-posedness which verifies $\lim _{f \rightarrow 0}[\Re(\beta(f)), \Im(\beta(f))]=(1,0)$.

5. Then, based on the LST results, the laminar HBL is excited at particular frequencies of interest $f$ by the means of a wall blowing mechanism. A $0.5 \mathrm{~mm}$ wide harmonic source of amplitude $v_{0}=$ $4.3 e^{-5} \times U_{\infty}=0.1 \mathrm{~m} . \mathrm{s}^{-1}$ is then centered at a pertinent location identified by the LST. It replaces the fully-reflective hard wall conditions at the corresponding wall flux points and simply reads as:

$$
W^{+}=W^{-}+2 \pi v_{0} f \cos (2 \pi f t) .
$$

6. Once the solution has converged, the oscillation amplitudes are measured and the HBL response is compared with the LST, for all the frequencies $f$ investigated, as to evaluate JAGUAR's capability in resolving the spatial growth rate.

7. The TDIBC is then activated in the region of interest identified by the LST. Once all transient waves have been washed out of the computational domain, the sound pressure level and other metrics of interest are sampled.

8. Finally, the previous two steps are repeated for the most unstable frequency with a reduced UAC surface in order to assess its impact on the pressure perturbation spatial growth and time-averaged solution as well.

\section{Precursor intake simulation (CEDRE)}

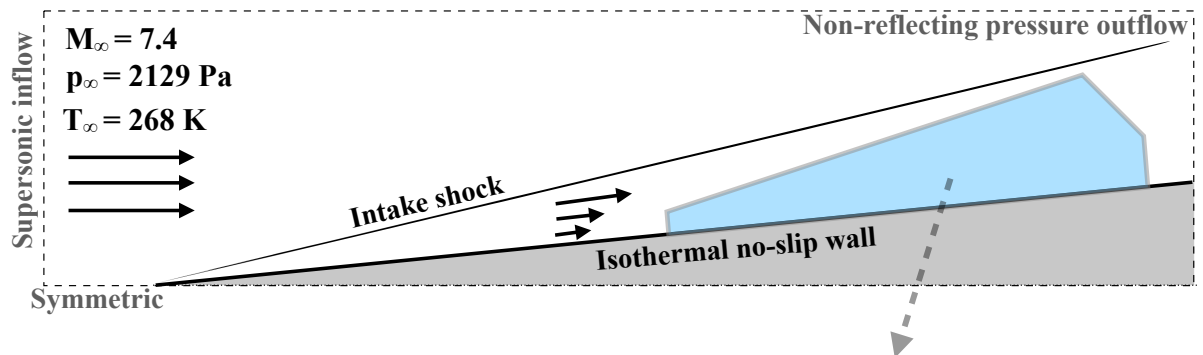

Hypersonic boundary layer DNS (JAGUAR)

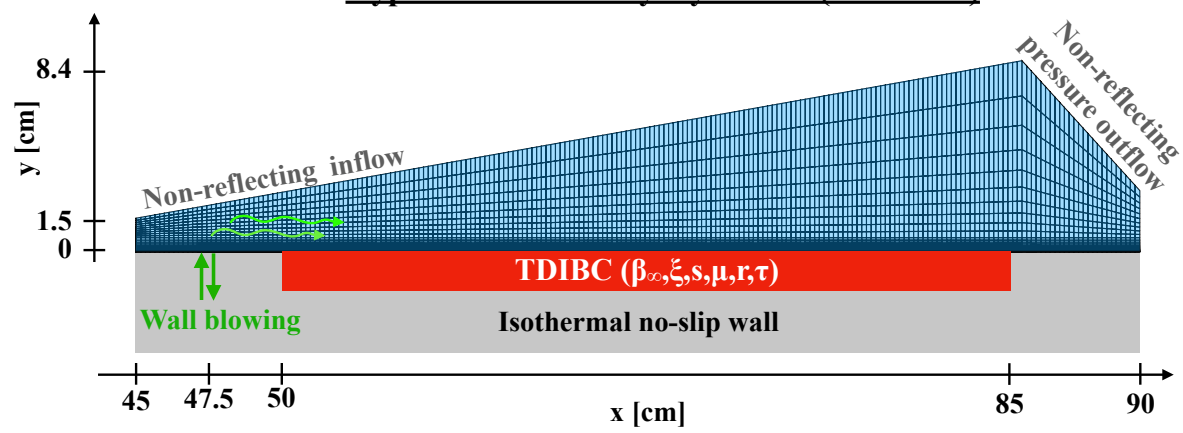

Figure 4. (Top) Configuration of the Mach 7.4 dihedral precursor simulation. (Bottom) Configuration of the HBL direct numerical simulation with excitation and UAC stabilization which uses characteristic boundary conditions calibrated from the precursor simulation. Only 1/24 mesh elements are displayed.

\section{B. Unperturbed direct numerical simulation of HBL}

The CEDRE precursor simulation is run over 10000 cycles of $1 \mu$ s each using a first-order implicit timeintegration scheme. The fluxes are evaluated using a second-order flux reconstruction scheme with a HLLC Riemann solver ${ }^{44}$ at the cell faces. The configuration is shown in Fig. 4. The mesh was generated with a streamwise spatial increment $\delta x=1 \mu \mathrm{m}$ at the leading edge and a 1.01 growth factor. Likewise, the cells at the walls have a size of $\delta y=1 \mu \mathrm{m}$ and a 1.04 growth factor. As a result, this $2 \mathrm{D}$ structured mesh is 
composed of approximately 2.2 million elements. The air is treated as a thermally perfect gas which uses a $7_{-}{ }^{\text {th }}$ order polynomial expression for the specific heat at constant pressure.

Once the solution has converged in time, the wall-normal velocity, temperature and pressure profiles at wall coordinate $x \in[0.2,1.2]$ are extracted into the LST solver MAMOUT. Further information about this solver are given in Esquieu et al. ${ }^{43,45}$ Excitation frequencies $f \in[180,200,220,240,260,280,300,360,420]$ $\mathrm{kHz}$. The resulting stability analysis is presented in Fig. 5 where the N-factors profiles are plotted for all frequencies along $x$. The $\mathrm{N}$-factor is defined as the natural logarithm of the pressure oscillation ratio, or as the integral of the exponential spatial growth rate $-\alpha_{i}$ of the most unstable mode. The following observations can be made. Frequencies lower than $180 \mathrm{kHz}$ are not expected to substantially destabilize the boundary layer within one meter of the leading edge. Frequencies higher than $420 \mathrm{kHz}$ will not cause a substantial growth anywhere in the HBL, with a maximum N-factor below 4 located around $30 \mathrm{~cm}$ from the leading edge. As a result, the region of interest to be investigated with the DNS was chosen to encompass the region $x \in[45,90] \mathrm{cm}$ where the limiting frequencies $f=180$ and $420 \mathrm{kHz}$ are inactive while all intermediate frequencies should experience partial growth.

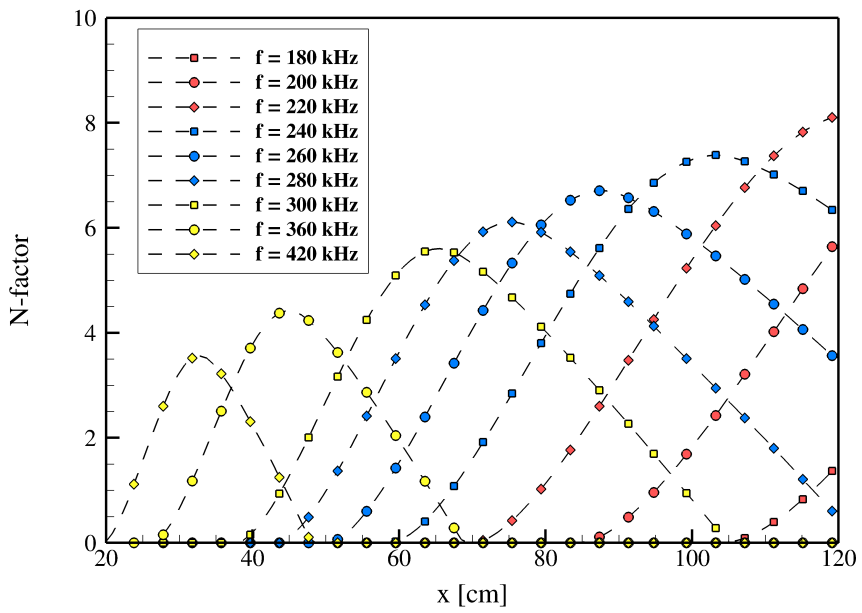

Figure 5. N-factor streamwise profiles obtained from the precursor simulation using linear stability theory.

Following step 3's instructions, as listed in Sec. A, a first unperturbed DNS is run using hard wall characteristic boundary conditions. It is initialized from the precursor simulation and uses its solution as well to impose the non-reflecting inflow/outflow boundary conditions. It uses the numerical methods presented in Sec. II with a $4^{t h}$-order SD method and $6^{\text {th }}$-stage Runge-Kutta explicit time-marching scheme. It ran for over half a millisecond until all transients were washed out using about 500 cpuh. The structured mesh is clustered as shown in Fig. 4 and uses $N_{x} \times N_{y}=900 \times 180=162000$ elements, or 2592000 SP. It possesses a uniform streamwise spatial increment of $0.5 \mathrm{~mm}$ and a first cell size at the wall of $10 \mu \mathrm{m}$. At the inlet plane, i.e. $\mathrm{x}=45 \mathrm{~cm}$, the boundary layer height $\delta$ equals $2.25 \mathrm{~mm}$ which results in a computational domain spawning $200 \delta$ and having at least 84 cells across the boundary layer. It is reminded that each cell is then further discretized into 9 solution points ( 3 along each direction). The air is again treated as a thermally perfect gas. Naturally, it uses the same $7^{\text {th }}$ order polynomial expression as the precursor simulation to evaluate the specific heat at constant pressure as a function of temperature.

The fourth step listed in Sec. A consists in activating the TDIBC and observe its passive effect on the unperturbed DNS. Based on the N-factor profiles, it was decided to start the TDIBC at $\mathrm{x}=50 \mathrm{~cm}$ (where the $260 \mathrm{kHz}$ unstable mode starts its growth) and end it at $\mathrm{x}=85 \mathrm{~cm}(5 \mathrm{~cm}$ before the computational domain end). The brutal activation of the TDIBC causes some weak oscillations of normal velocity at the wall which quickly damp as the boundary condition equilibrates. Once the solution has stabilized, the solutions from all three simulations ran up to this point are compared. Figures 6 and 7 present various wall-normal profiles of, respectively, the streamwise $u$ and wall-normal $v$ instantaneous (as opposed to time-averaged) velocities for all three cases. Interestingly, while the streamwise profiles appear to collapse on top of each other, this is not the case for the weak $v$ profiles. In the latter case, the precursor simulation presented slightly higher amplitude profiles. This is caused by weak a discontinuity which invariably appears at the sonic point located on the inlet plane in all DNS. The unperfect inflow conditions are responsible for the inevitable appearance 
of this artificial shocklet (pressure jump inferior to 5 Pascal). In response, the flow is slightly deflected away from the wall, which triggers a weak expansion affecting the velocity profiles. This is further seen in Fig. 8 which compares the wall pressure profiles. Indeed, the differences between the DNS and the precursor solution appear to diminish further away from the inlet. On the other hand, both DNS solutions appear to match throughout the computational domain. Importantly, this validates the low-frequency passivity of the TDIBC. Overall, the discrepancies between all cases are deemed to be sufficiently small to render relevant DNS/LST comparisons.
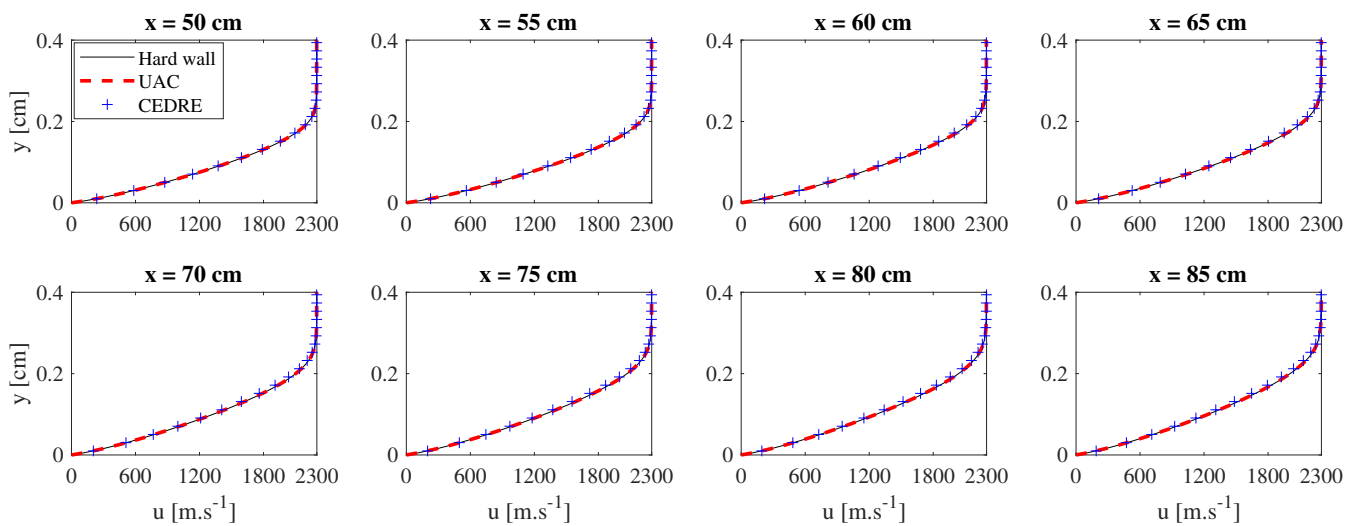

Figure 6. Comparison of instantaneous wall-normal profiles of unperturbed streamwise velocity at various streamwise locations across the hypersonic boundary layer.
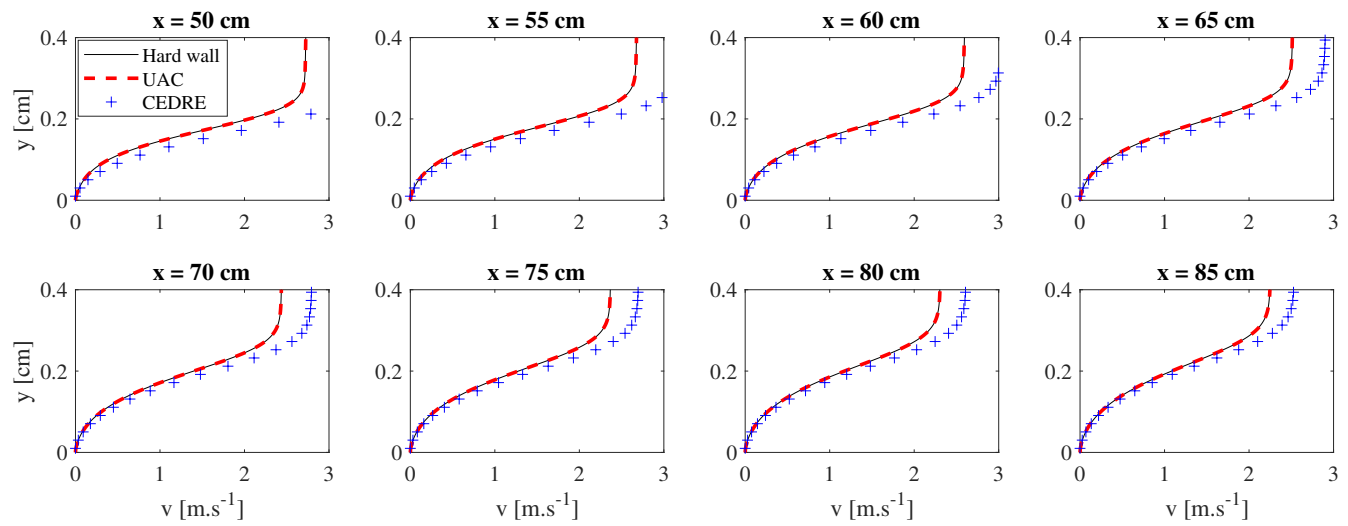

Figure 7. Comparison of instantaneous wall-normal profiles of unperturbed wall-normal velocity at various streamwise locations across the hypersonic boundary layer.

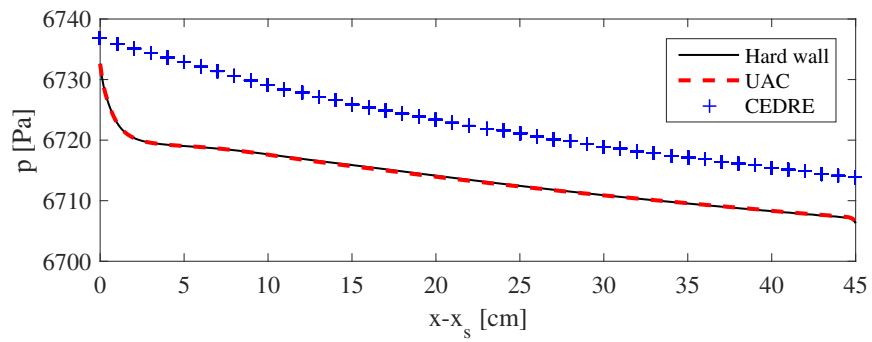

Figure 8. Comparison of unperturbed instantaneous wall static pressure profiles along the wall starting at $x_{s}$ $=45 \mathrm{~cm}$.

\section{Excitation and stability analysis}

Having verified the coherence of the DNS unperturbed flow with the precursor solution, a source is then placed along the wall as discussed in Sec. A (step 5). It is located at $\mathrm{x}=47.5 \mathrm{~cm}$, right upstream of the 
$260 \mathrm{kHz}$ unstable mode's growth region. The HBL response to the following frequencies is now investigated: $f \in[180,200,220,240,260,280,300,420] \mathrm{kHz}$.

As the source activates, a strong transient response is observed in all cases. It is wonderfully revealed by plotting the wall pressure envelope signals in the space-time presented in Figs. 9 and 10 for the 180 and 300 $\mathrm{kHz}$ excitation frequencies, respectively. These space-time diagram are built from 451 wall static pressure probes placed every millimeter from $x-x_{s}=0$ to $45 \mathrm{~cm}$, where $x_{s}=45 \mathrm{~cm}$ is the distance of the DNS inlet plane from the leading edge. First, the $180 \mathrm{kHz}$ case's envelope presents a wave-packet format which indicates a nonlinear dynamic response to the initial perturbation. Eventually, this wave-packet convects through space at the constant speed of sound and exits the computational domain. Interestingly, the pressure oscillation quickly dampens after its passage at every $x$-station: this confirms the LST results which predicted the $x \in[45,90]$ region to be stable under $180 \mathrm{kHz}$ perturbations. Strikingly, this is not the case for the 300 $\mathrm{kHz}$ case: not only is the wall static pressure envelop growing in space, but strong oscillations remain once a permanent response has been established across the computational domain.

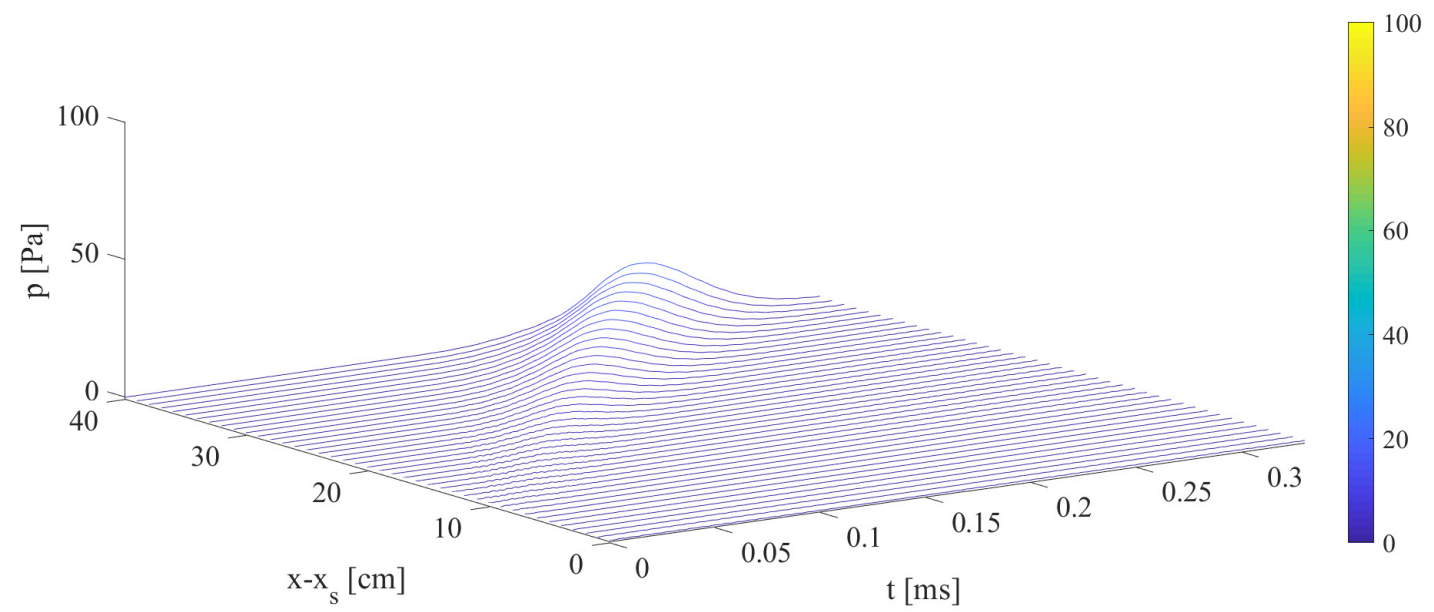

Figure 9. Space-time envelop of a wall static pressure $180 \mathrm{kHz}$-perturbation introduced at $x-x_{s}=2.5 \mathrm{~cm}$ as it excites the boundary layer (colored by static pressure $p[\mathrm{~Pa}]$ ).

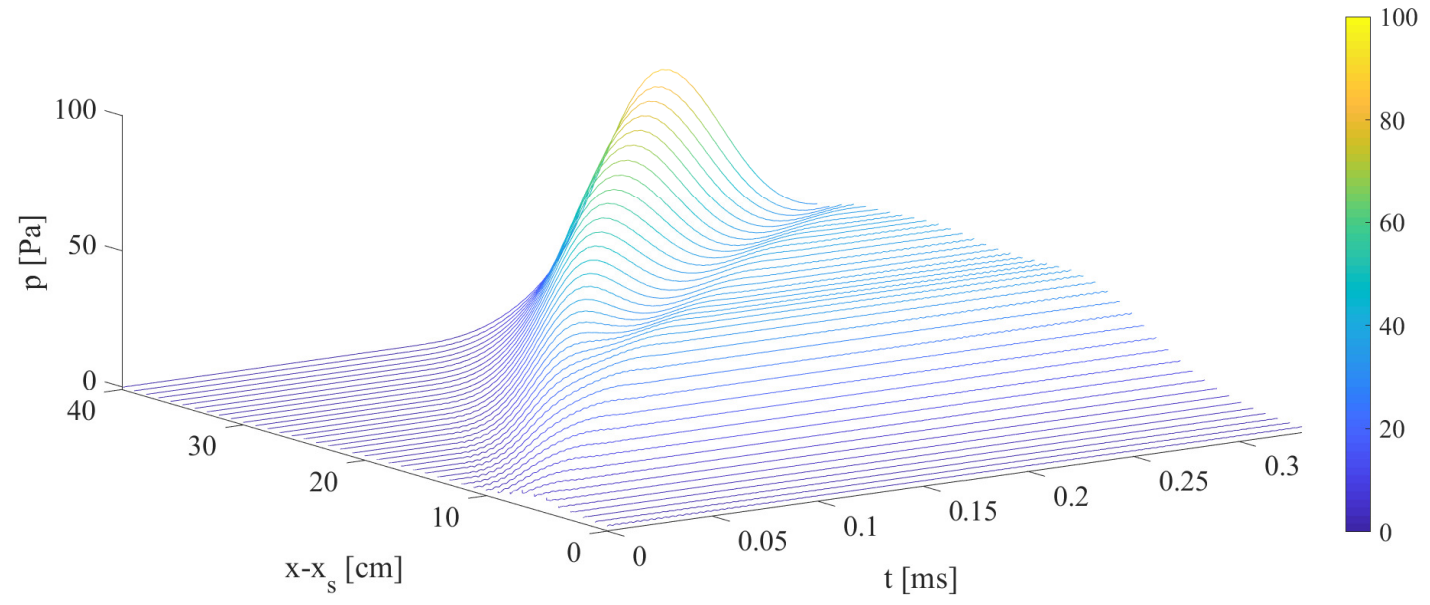

Figure 10. Space-time envelop of a wall static pressure $300 \mathrm{kHz}$-perturbation introduced at $x-x_{s}=2.5 \mathrm{~cm}$ as it excites the boundary layer (colored by static pressure $p[\mathrm{~Pa}]$ ).

The LST predicted that the $260 \mathrm{kHz}$ case would, amongst the interrogated frequencies, be the most unstable throughout the region spawned by the DNS. The static pressure contours should, therefore, be mostly responsive to its perturbations. Figure 11 presents snapshots of static pressure perturbations $p^{\prime}$ illustrating the strong growth of waves by many orders of magnitudes over a few tens of centimeters only. Pressure oscillations also appear around the sonic line which is often observed and indicative of secondmode transition $^{27,46}$ Finally, Fig. 12 quantifies the growth of wall pressure perturbations and provides a 
comparison with the previously discussed LST results. A superposition of the N-factor plots shown in Fig. 5 with the profiles obtained from the DNS offers little insight (left figure) as the DNS perturbations are only initiated at $x-x_{s}=2.5 \mathrm{~cm}$. Hence, a quick rescaling along the y-axis is necessary, and reveals how the profiles collapse remarkably well despite the small discrepancies shown in Fig. 8. It can be noted that the $420 \mathrm{kHz}$ is not plotted in this figure: it was observed to naturally decay and never cross the N-factor positive plane. This is an important feature predicted by the LST that the DNS was able to capture. A comparison of wall-normal pressure perturbation amplitude profiles shown in Fig. 13 for the $260 \mathrm{kHz}$ excitation case further highlights the good agreement between the solvers. Importantly, all these results are a testimony to the DNS capability in mirroring the LST growth rates for the hard wall case. This opens up the way towards the study's next step: perturbations damping through the use of the TDIBC.
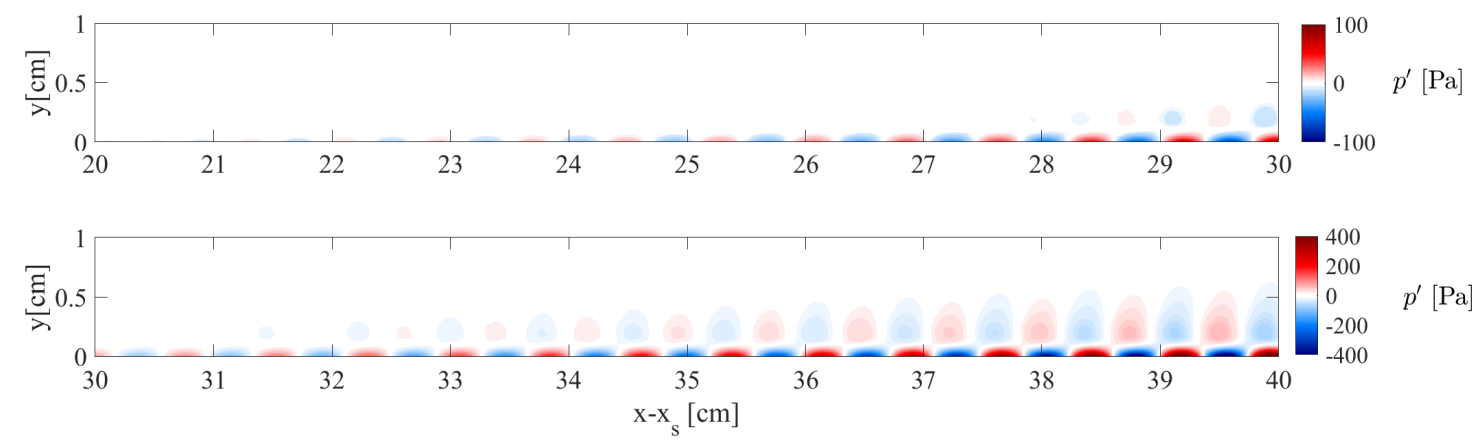

Figure 11. Static pressure perturbations snapshots for (top) $x \in x_{s}+[20,30]$ and (bottom) $x \in x_{s}+[30,40]$ for the hard wall with a $260 \mathrm{kHz}-e x c i t a t i o n$ case.
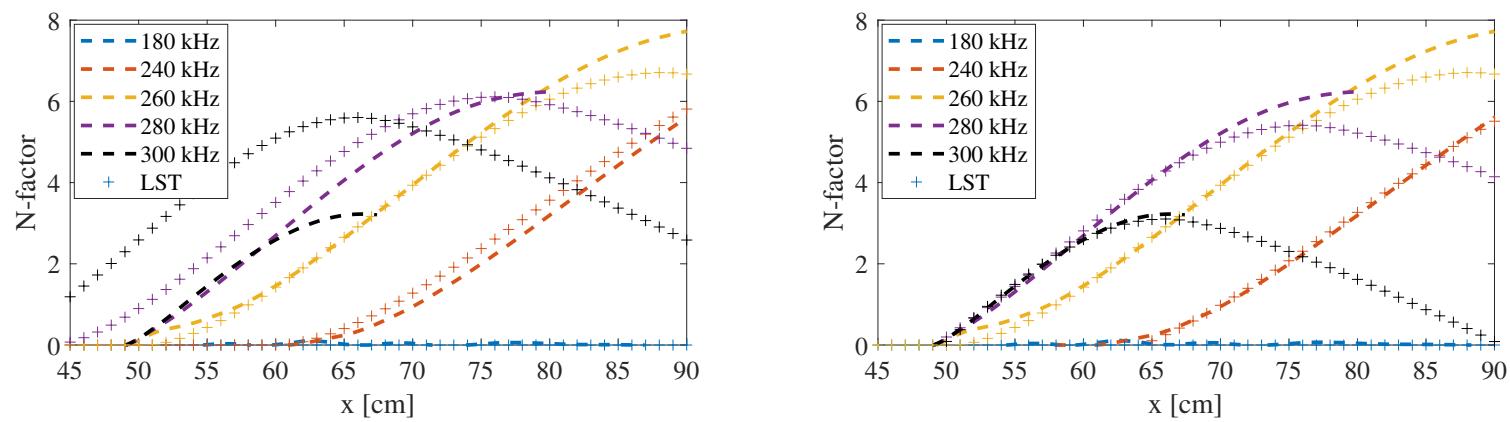

Figure 12. (Left) Gross and (right) rescaled N-factor profiles for various excitation frequencies obtained from the (+) LST and from the (dotted) DNS.

\section{Analysis of the stabilizing effect of the UAC}

The TDIBC presented in Sec. III is now activated and is initially located as shown in Fig. 4. It is calibrated to replicate the broadband response of the DLR's UAC, as was previously explained.

\section{A. Suppression of the wall pressure oscillations by the UAC}

Just as the activation of the source term in the HBL marked the start of a transient regime (see Figs. 9 and 10), the same occurred when activating the TDIBC of the UAC. This time however, its effect was a strong and quick suppression of the instabilities as seen in Fig. 14 for the $260 \mathrm{kHz}$ case with the wall pressure oscillation amplitude at the outlet plane being resolutely damped.

The stabilizing effect is further studied for the $260 \mathrm{kHz}$ case of interest by looking at the power spectral density of the wall pressure perturbations at $x-x_{s}=4,45 \mathrm{~cm}$, which correspond to positions upstream/downstream of the TDIBC. They are respectively shown in Figs. 15 and 16. Importantly, the TDIBC has no recursive effect on the upstream source, which would have raised questions on the pertinence of a direct comparison between the TDIBC and hard wall cases. The power spectral density peak value has been decreased by 6 orders of magnitude: this corresponds to a decrease in wall pressure oscillation 

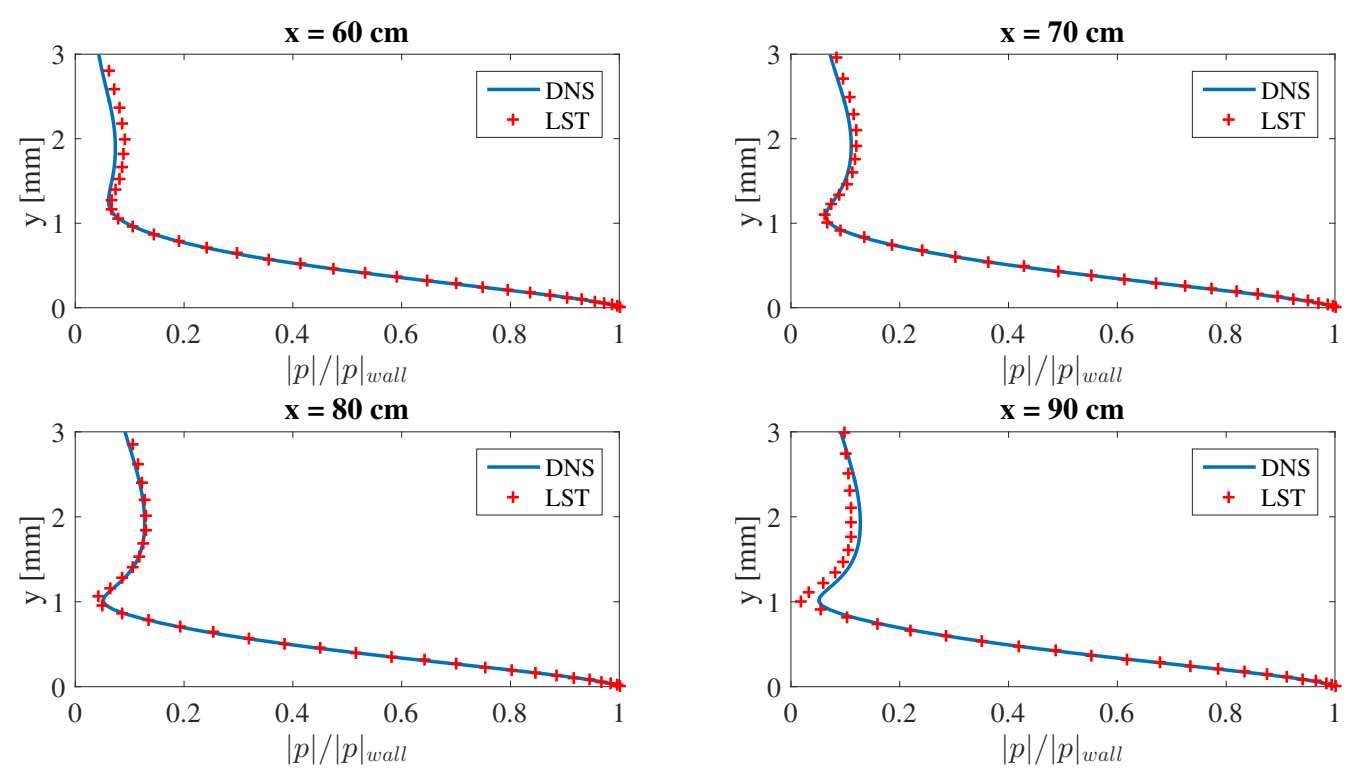

Figure 13. Wall-normal pressure perturbations amplitude at different streamwise locations for the LST and DNS solutions when using a hard wall boundary condition.

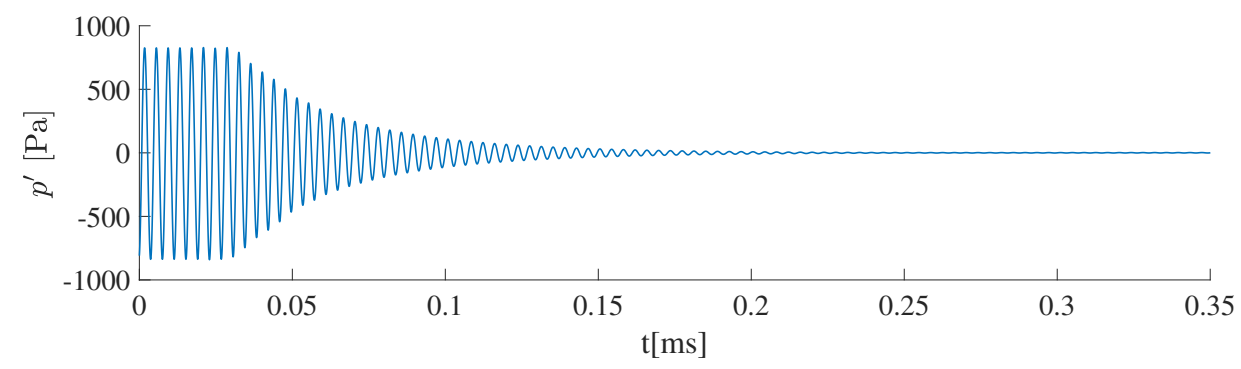

Figure 14. Wall pressure time-signal at $x-x_{s}=45 \mathrm{~cm}$ during the activation of the UAC numerical boundary condition.

amplitude by a factor 1000. Similar damping is observed for the other frequencies as presented in Fig. 17, albeit the amplitude of the pressure perturbation has not grown as much as the with the $260 \mathrm{kHz}$ excitation. Notably, the limiting 180 and $420 \mathrm{kHz}$ excitation cases result in a similarly weak wall pressure power spectral density at the domain's outlet, being outside the range of unstable frequency in this particular region as predicted by the LST.

The impact of the UAC on the instability growth is, once again, quantified by evaluating the N-factor along the streamwise direction. These are plotted in Fig. 18 for the same frequencies shown in Fig. 12. Once again, the $420 \mathrm{kHz}$ does not cross into the positive $\mathrm{N}$-factor plane and was not plotted for clarity purpose. The left figure directly compares the profiles with and without UAC, while the right figure shows the difference between these profiles. This difference corresponds to the integration in space of a $\Delta \alpha_{i, \mathrm{UAC}}$ directly corresponding to the UAC impact on the pressure growth rate. Interestingly, these profiles appear strikingly linear and almost parallel across the TDIBC region which is reminded to be $x \in[50,85]$. This indicates that the stabilization factor the UAC provides is roughly equivalent to a constant exponential pressure growth rate $\alpha_{i, \mathrm{UAC}}=-20$. This is very encouraging as it indicates that the TDIBC, homogeneously defined in space and time by its poles and weights (see Tab. 2), also presents a homogeneous effect on the growth rate of unstable modes. However, more surprisingly, it is observed to be practically independent of the frequency for the $[180,300] \mathrm{kHz}$ range. A glance at the UAC broadband properties (see Fig. 2) confirms a relatively constant reflectivity bounded between 0.55 and 0.65 around the first anti-resonance for this frequency range. This could explain the aforementioned low spread in damping coefficients. 

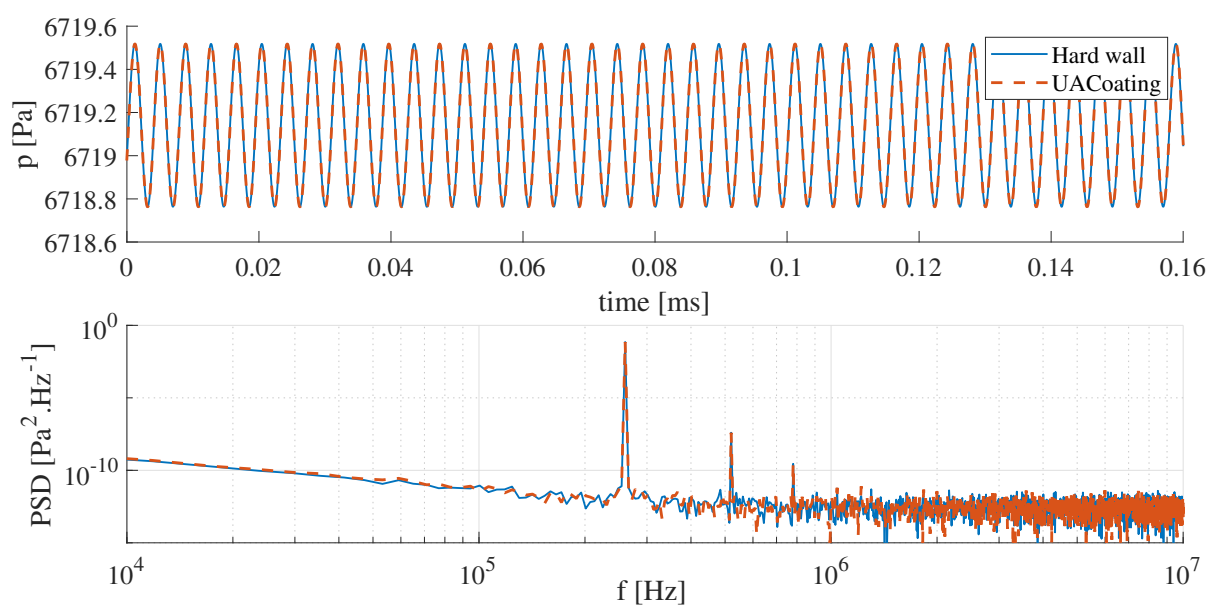

Figure 15. (Top) Wall pressure time-signals at $x-x_{s}=4 \mathrm{~cm}$ and (bottom) their corresponding power spectral densities for the $260 \mathrm{kHz}$-excitation case over the hard and ultrasonically absorbent walls.
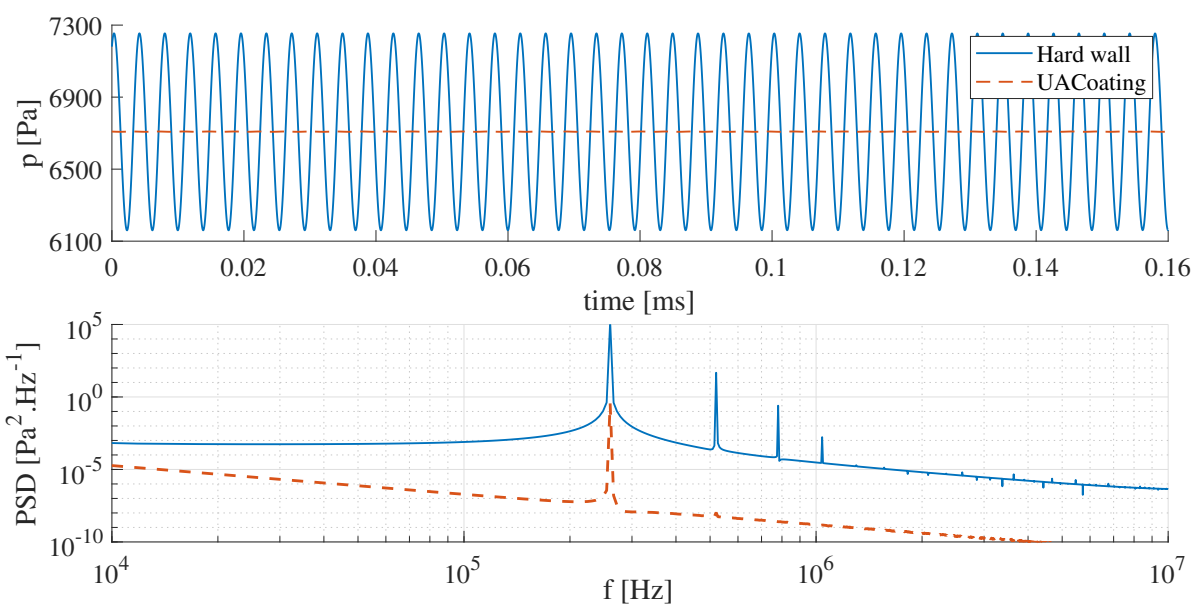

Figure 16. (Top) Wall pressure time-signals at $x=85 \mathrm{~cm}$ and (bottom) their corresponding power spectral densities for the $260 \mathrm{kHz}-$ excitation case over the hard and ultrasonically absorbent walls.

\section{B. Activation of supersonic mode and radiation of noise}

The effect of the UAC on the static pressure field further away from the wall is illustrated in Fig. 19 which corresponds to the contours shown in Fig. 11 once the TDIBC has been activated and all transient washed out. It can be noticed that, besides the contour's bounds decreasing by two orders of magnitude, there appears to be some pressure wave radiation towards the far-field. It originates from within the boundary layer and these waves were not present in the hard wall case. According to Knisely et al., ${ }^{10,11}$ the subsidence of pressure oscillations further away from the wall suggests that a fast supersonic mode might become dominant over the slow acoustic mode. This would suggest that the UAC is particularly effective at suppressing the slow mode and less so with the higher ones.

Having observed the effect the UAC has on the acoustic far-field, one wonders if it also has any impact on the time-averaged static pressure contours. These are now scrutinized for the hard wall and UAC- $260 \mathrm{kHz}$ excitation cases: they are presented, respectively, in Figs. 20 and 21. First, it is reminded that the $260 \mathrm{kHz}$ excitation hard wall case resulted in pressure perturbations on the order of hundreds of Pascals (see Fig. 11). Yet, there appears to be no mark of these strong oscillations on the time-averaged contours, as it should be for a linear super-imposed process. On the other hand, the case with the TDIBC exhibits strong oscillations attached to the wall whose wavelength matches the $260 \mathrm{kHz}$ excitation frequency. Surprisingly, the amplitude of these time-averaged oscillations are also much higher than the instantaneous perturbations shown in Fig. 19. Again, it is reminded that the unperturbed solution using the UAC and hard wall numerical boundary 

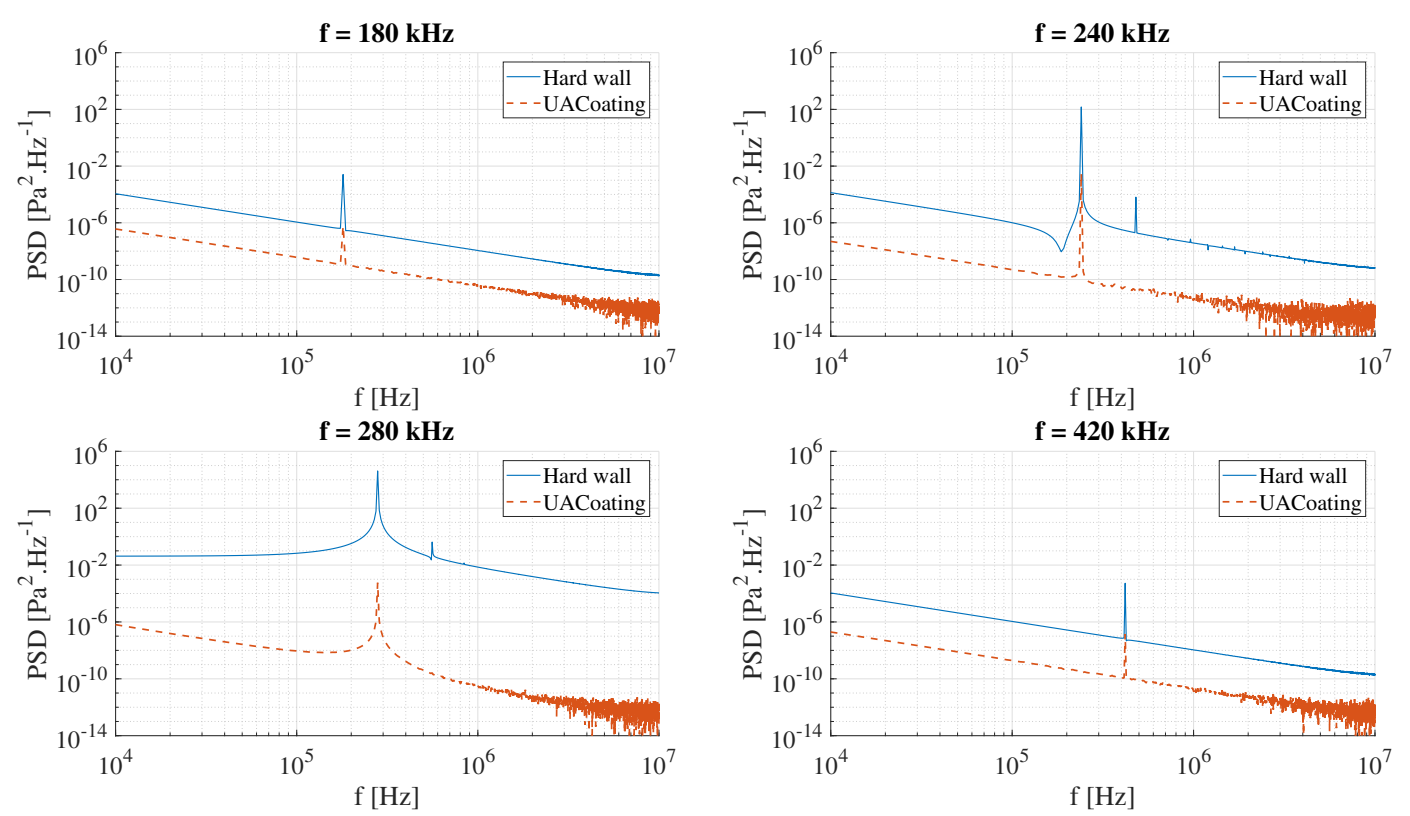

Figure 17. Power spectral densities of the $x=85 \mathrm{~cm}$ wall pressure signals for various excitation frequencies over the hard and ultrasonically absorbent walls.
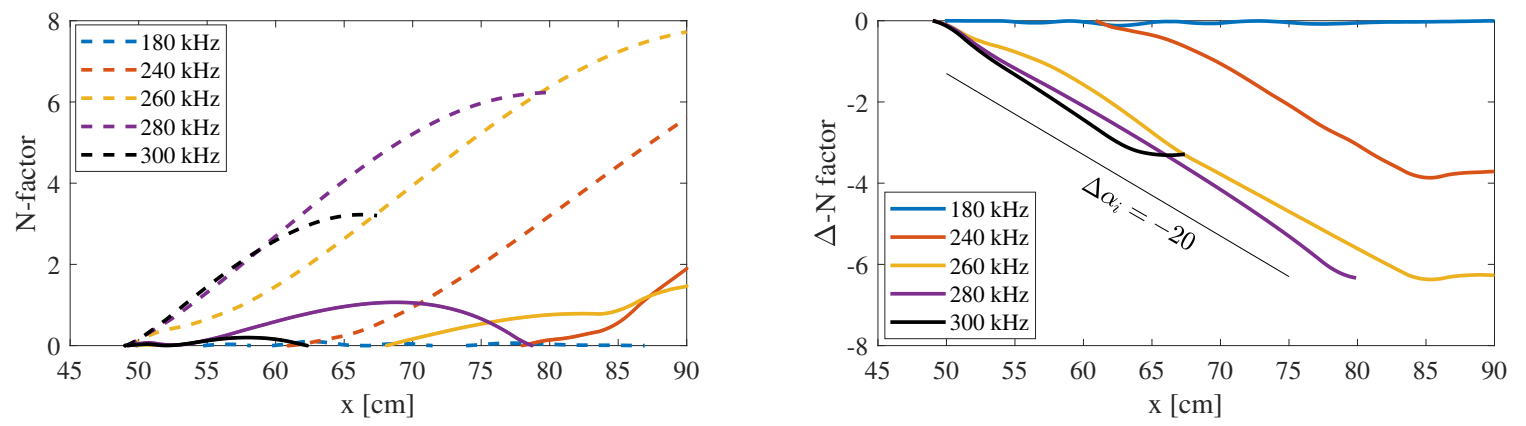

Figure 18. (Left) N-factor profiles for the (dashed) hard and (solid) ultrasonically-absorbent walls. (Right) Stabilizing impact of the UAC on the wall pressure growth rate.
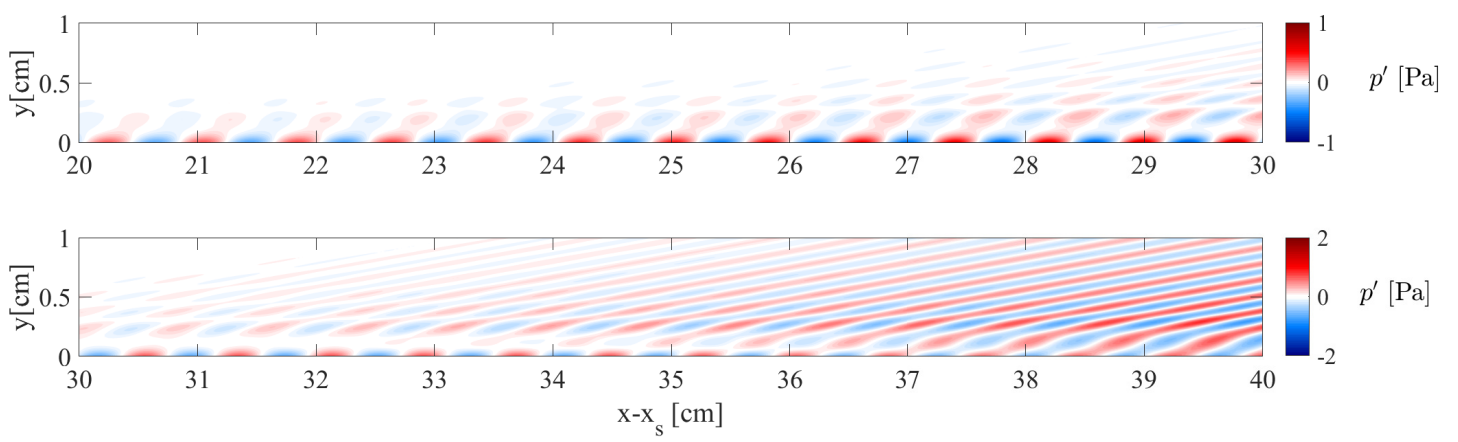

Figure 19. Static pressure perturbations snapshots for (top) $x \in x_{s}+[20,30]$ and (bottom) $x \in x_{s}+[30,40]$ for the UAC wall with a $260 \mathrm{kHz}-$ excitation case.

conditions were identical. Therefore, the excited UAC has triggered a strong nonlinear response from the instantaneous and mean flow altogether.

To conclude, a particular trade-off between enhanced stability and noise has been found. It is due to the UAC radiating noise under excitation, amounting to a sort of wall singing phenomenon, and to the formation of high-amplitude standing waves attached to the UAC. 

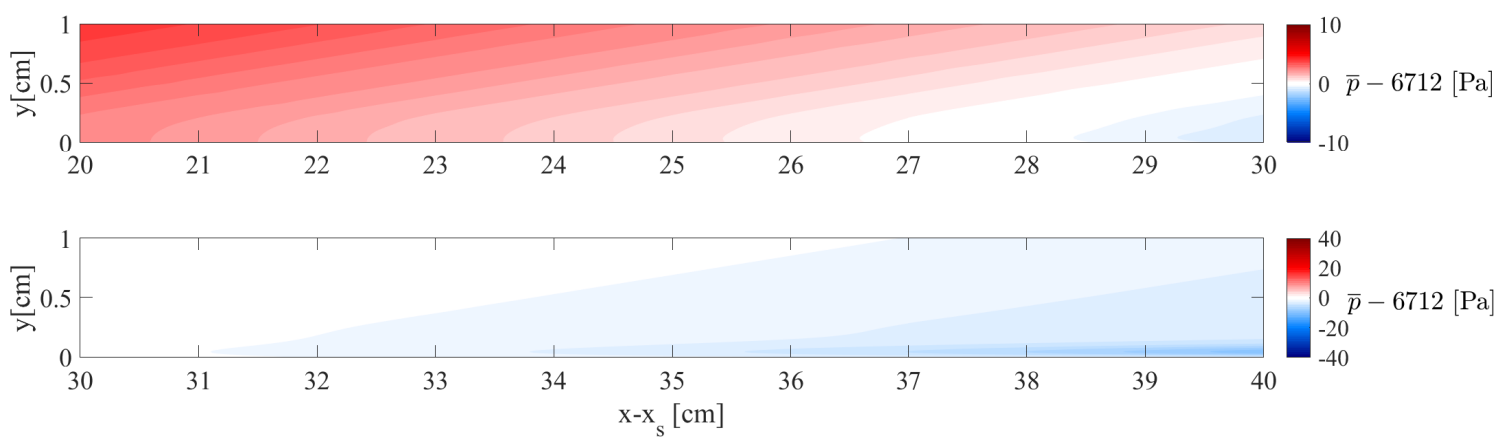

Figure 20. Time-averaged contours $\bar{p}-6712[\mathrm{~Pa}]$ for (top) $x \in x_{s}+[20,30]$ and (bottom) $x \in x_{s}+[30,40]$ for the hard wall with a $260 \mathrm{kHz}$-excitation case.
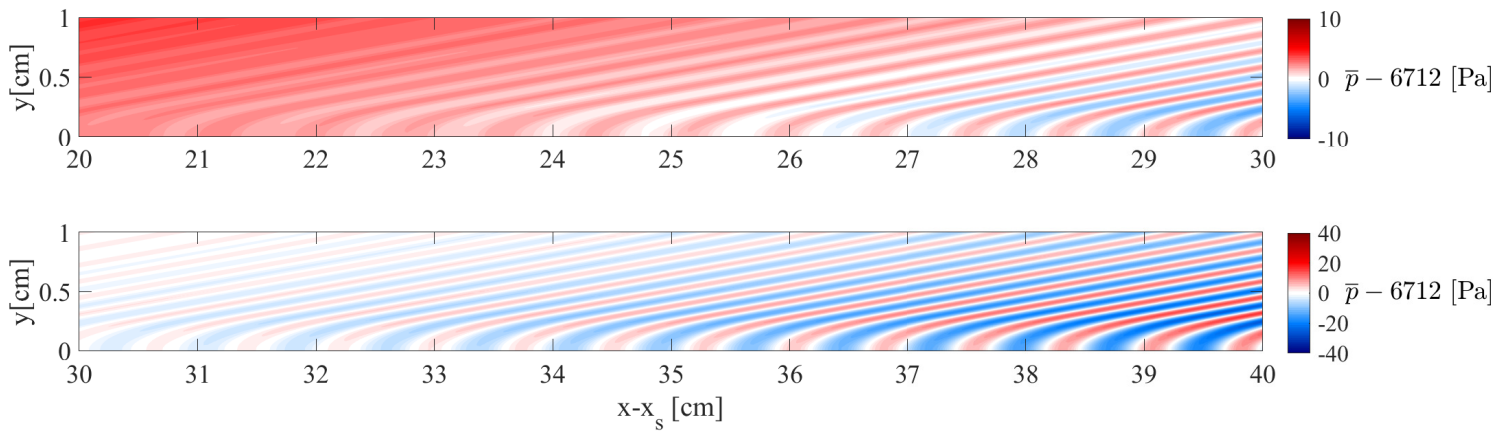

Figure 21. Time-averaged contours $\bar{p}-6712[\mathrm{~Pa}]$ for (top) $x \in x_{s}+[20,30]$ and (bottom) $x \in x_{s}+[30,40]$ for the UAC with a $260 \mathrm{kHz}-$ excitation case.

\section{Effect of the UAC positioning}

The $260 \mathrm{kHz}$ case was further studied by investigating the effect of displacing the TDIBC further downstream on the suppression of the second-mode instabilities. Three new test cases are run where the TDIBC starts at $x$ $=60,70,80 \mathrm{~cm}$ instead of $50 \mathrm{~cm}$. The end location is kept at $\mathrm{x}=85 \mathrm{~cm}$. This sensitivity study is motivated by the need to understand how much surface has to be treated to achieve a target reduction of HBL instabilities. Coincidently, new LST calculations are also carried at $260 \mathrm{kHz}$ which use the constant impedance resistivity and reactivity calculated from Sousa et al. ${ }^{26}$ at this particular frequency. This permits to evaluate the LST ability in locally resolving these complex flows on its own. The N-factor profiles from the DNS and LST for all configurations are presented in Fig. 22. First, all DNS and LST seem to react similarly near the TDIBC edge, independently of its placement. Second, the longer the UAC, the stronger the suppression of instability waves. Third, the LST and DNS solutions appear to diverge and the discrepancies appear to build in space. This is probably due to the modification of the time-averaged supporting pressure field by the TDIBC observed in the DNS. The LST is not able to resolve this nonlinear response and over-predicts the suppression capabilities of the UAC.

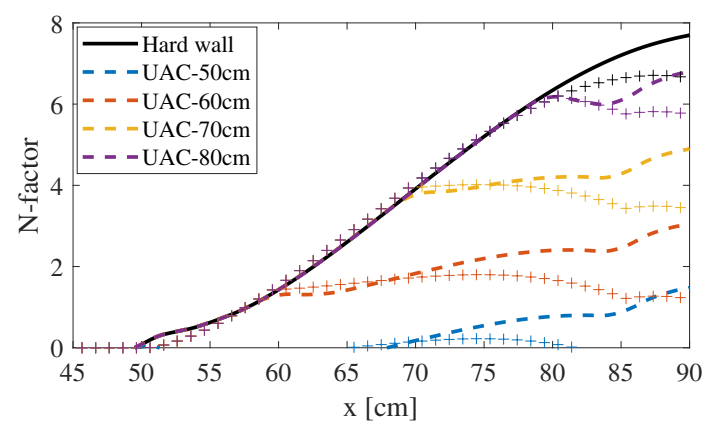

Figure 22. N-factor profiles for the $260 \mathrm{kHz}$-perturbation case for various wall boundary conditions using the (+) LST and the (dashed) DNS. 
Finally, this study concludes by observing the impact the UAC placement has on the perturbations further away from the wall. To this end, the $x=90 \mathrm{~cm}$ wall-normal profiles of pressure oscillation amplitudes, $\tilde{p}$ $[\mathrm{dB}]$ are plotted in Fig. 23. This corresponds to the outlet plane, $5 \mathrm{~cm}$ after the end of any TDIBC. As expected, the longest the UAC, the more suppressed the oscillations are close to the wall. However, further away, there does not appear to be a linear relation between UAC length and radiation noise levels. In fact, placing a small portion of UAC later downstream once the instabilities have already grown considerably tends to backfire, as is done with the UAC- 80 case. This suggests that further studies are needed to optimize the use of UAC for passive control is hypersonic boundary layers.

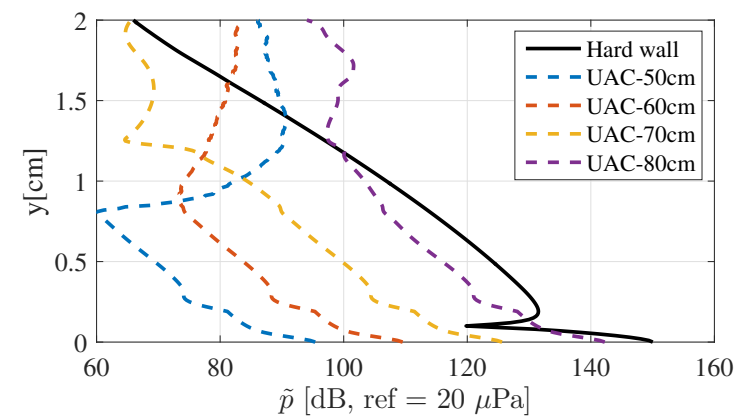

Figure 23. Wall-normal profile of pressure perturbations measured in $\mathrm{dB}$ at $\mathrm{x}=90 \mathrm{~cm}$ (outlet plane) for various UAC positions.

\section{Conclusions}

The second-mode stabilizing capacity of ultra-sonically absorbent coatings for hypersonic boundary layers has been studied by the means of direct numerical simulations.

These high-fidelity simulations used a high-order spectral difference method, which is unprecedented for such applications to the author's best knowledge. This numerical scheme is able to retain a high-order of accuracy on unstructured meshes, hence its importance in simulating complex geometries. Until recently, however, this family of schemes was missing proper boundary conditions required to resolve aeroacoustics problem. Notably, non-reflecting boundary conditions required to prevent spurious reflections from polluting the inner domain acoustic were only recently implemented with SD. ${ }^{31}$ Coincidently, time-domain impedance boundary conditions recently derived by Monteghetti et al. ${ }^{30}$ proven to be broadband-accurate, were also enabled with SD. ${ }^{31}$ The conjunction of both types of boundary conditions has resulted in the development of a high-order numerical flow solver aimed at investigating the underlying physics of hypersonic boundary-layer transition and eventually designing UAC-based passive control devices.

Specifically, the solver ability in resolving the growth rate of unstable mode was demonstrated through comparison with linear stability theory. The use of UAC, modeled through TDIBC, was also proven to be a formidable passive control device and promising technology. Further, the suppression of second-mode instability was observed to coincidentally trigger the radiation of sound outside the boundary layer.

These results highlight the need to further develop numerical tools to guide the design of optimal coatings mitigating the trade-off. To this end, the implementation of adjoint-based optimization tools closely coupled with these high-order methods are the subject of ongoing work. Overall, this seminal study is a testament to the maturity this integrated numerical platform, JAGUAR, has reached. It serves as a milestone towards the achievement of the aforementioned long-term objectives.

\section{Acknowledgement}

The first author is funded by the STAE foundation (Science and Technology for Aeronautics and Space), grant number CDT-R050-L00-T00. This work is part of the STAE-funded 3C2T project (control of compressible, transitional and turbulent boundary layer) involving ONERA, CERFACS and the IMFT under the supervision of Christophe Airiau. The authors also thank Loï Jecker for his kind help in configuring the precursor simulation using the code CEDRE. 


\section{Appendix}

The table below presents all the scalars used to discretize the TDIBC matching the broadband profile shown in Fig. 2.

\begin{tabular}{|c|c|c|}
\hline \hline Scalars & $\beta_{\infty}=8.187879 e^{-1}$ & $\tau=5.877685 e^{-6} \mathrm{~s}$ \\
\hline Poles & \multicolumn{2}{|c|}{ Functions weights } \\
\hline$\left(\tilde{s}_{n},-\tilde{\xi}_{k}\right)_{n, k}\left(\mathrm{rad} . \mathrm{s}^{-1}\right)$ & $\left(\tilde{r}_{1, n},-\tilde{\mu}_{1, k}\right)_{n, k}\left({\left.\mathrm{rad} . \mathrm{s}^{-1}\right)}_{1}\right.$ & $\left(\tilde{r}_{2, n},-\tilde{\mu}_{2, k}\right)_{n, k}\left({\left.\mathrm{rad} . \mathrm{s}^{-1}\right)}^{-1}\right.$ \\
\hline$-1.256637 \mathrm{e}+3$ & $5.660893 \mathrm{e}+0$ & $4.277775 \mathrm{e}+0$ \\
\hline$-2.148819 \mathrm{e}+4$ & $6.807834 \mathrm{e}+2$ & $9.184530 \mathrm{e}+2$ \\
\hline$-3.674429 \mathrm{e}+5$ & $3.589210 \mathrm{e}+4$ & $-4.261821 \mathrm{e}+4$ \\
\hline$-6.283185 \mathrm{e}+6$ & $-1.331916 \mathrm{e}+6$ & $-417427 \mathrm{e}+4$ \\
\hline$(-1.974050 \mathrm{e}+5,3.1685357 \mathrm{e}+5)$ & $(-5.737098 \mathrm{e}+4,-8.382613 \mathrm{e}+3)$ & $(1.115722 \mathrm{e}+4,-2.097452 \mathrm{e}+4)$ \\
\hline$(-3.544161 \mathrm{e}+2,1.234667 \mathrm{e}+6)$ & $(-5.028205 \mathrm{e}+4,-1.018499 \mathrm{e}+4)$ & $(-4.218533 \mathrm{e}+3,-9.128232 \mathrm{e}+3)$ \\
\hline$(-4.614602 \mathrm{e}+5,2.198411 \mathrm{e}+6)$ & $(-5.601881 \mathrm{e}+4,-1.478211 \mathrm{e}+4)$ & $(-4.509185 \mathrm{e}+3,-2.663290 \mathrm{e}+3)$ \\
\hline$(-5.482225 \mathrm{e}+5,3.181595 \mathrm{e}+6)$ & $(-6.548491 \mathrm{e}+4,-1.802480 \mathrm{e}+4)$ & $(-2.827210 \mathrm{e}+3,-2.504131 \mathrm{e}+2)$ \\
\hline$(-6.231411 \mathrm{e}+5,4.176283 \mathrm{e}+0)$ & $(-8.044434 \mathrm{e}+4,-1.886116 \mathrm{e}+4)$ & $(-1.356928 \mathrm{e}+3,2.770425 \mathrm{e}+2)$ \\
\hline$(-6.900477 \mathrm{e}+5,5.178806 \mathrm{e}+6)$ & $(-1.101268 \mathrm{e}+5,-1.467016 \mathrm{e}+4)$ & $(-3.609999 \mathrm{e}+2,-1.731994 \mathrm{e}+2)$ \\
\hline$(-7.510679 \mathrm{e}+5,6.187110 \mathrm{e}+6)$ & $(-2.339126 \mathrm{e}+5,-2.490394 \mathrm{e}+4)$ & $(1.160141 \mathrm{e}+3,-2.148801 \mathrm{e}+3)$ \\
\hline$(-8.075265 \mathrm{e}+5,7.199905 \mathrm{e}+6)$ & $(-5.691639 \mathrm{e}+4,-5.317827 \mathrm{e}+5)$ & $(6.532230 \mathrm{e}+3,3.590720 \mathrm{e}+3)$ \\
\hline \hline
\end{tabular}

Table 2. Scalars defining the ODR representation of the UAC presented in Fig. 2.

\section{References}

${ }^{1}$ Reed, H., Kimmel, R., Schneider, S., and Arnal, D., "Drag prediction and transition in hypersonic flow, AIAA Paper 1997-1818," AIAA 28th Fluid Dynamics Conference, 1997.

${ }^{2}$ Zhong, X. and Wang, X., "Direct numerical simulation on the receptivity, instability, and transition of hypersonic boundary layers," Annual Review of Fluid Mechanics, Vol. 44, 2012, pp. 527-561.

${ }^{3}$ Fedorov, A., "Transition and stability of high-speed boundary layers," Annual Review of Fluid Mechanics, Vol. 43, 2011, pp. 79-95.

${ }^{4}$ Malik, M., Zang, T., and Bushnell, D., "Boundary layer transition in hypersonic flows, AIAA Paper 90-5232," 2nd AIAA International Aerospace Planes Conference, Orlando, October 1990.

${ }^{5}$ Mack, L., "Review of linear compressible stability theory," Stability of Time Dependent and Spatially Varying Flows, edited by D. Dwoyer and M. Hussaini, ICASE NASA LaRC, Springer, 1987, pp. 164-187.

${ }^{6}$ Morkovin, M. V., Transition at hypersonic speeds, NASA Langley Research Center, Hampton, VA, United States, 1987.

${ }^{7}$ Mack, L., "On the inviscid acoustic-mode instability of supersonic shear flows. Part 1: two-dimensional waves," Theoretical and Computational Fluid Dynamics, Vol. 2, No. 2, 1990, pp. 97-123.

${ }^{8}$ Egoroc, I. V., Fedorov, A. V., and Soudakov, V. G., "Receptivity of a hypersonic boundary layer over a flat plate with a porous coating," Journal of Fluid Mechanics, Vol. 601, 2008, pp. 165-187.

${ }^{9} \mathrm{Li}, \mathrm{X} ., \mathrm{Fu}, \mathrm{D} .$, and Ma, Y., "Direct Numerical Simulation of Hypersonic Boundary Layer Transition over a Blunt Cone," AIAA Journal, Vol. 46, No. 11, 2008, pp. 2899-2913.

${ }^{10}$ Knisely, C. P. and Zhong, X., "Sound radiation by supersonic unstable modes in hypersonic blunt cone boundary layers. I. Linear stability theory," Physics of Fluids, Vol. 31, No. 2, 2019, pp. 024103.

${ }^{11}$ Knisely, C. P. and Zhong, X., "Sound radiation by supersonic unstable modes in hypersonic blunt cone boundary layers. II. Direct numerical simulation," Physics of Fluids, Vol. 31, No. 2, 2019, pp. 024104.

${ }^{12}$ Kuehl, J. J., "Thermoacoustic Interpretation of Second-Mode Instability," AIAA Journal, Vol. 56, No. 9, 2018, pp. 35853592.

${ }^{13}$ Mack, L., "Effect of cooling on boundary-layer stability at Mach number 3," Instabilities and Turbulence in Engineering Flows, edited by D. Ashpis, T. Gatski, and R. Hirsh, Springer, 1993, pp. 175-188.

${ }^{14}$ Fedorov, A., Malmuth, N., Rasheed, A., and Hornung, H., "Stabilization of hypersonic boundary layers by porous coatings," AIAA Journal, Vol. 39, No. 4, 2001, pp. 605-610.

${ }^{15}$ Rasheed, A., Hornung, H., Fedorov, A., and Malmuth, N., "Experiments on passive hypervelocity boundary-layer control using an ultrasonically absorptive surface," AIAA Journal, Vol. 40, No. 3, 2002, pp. 481-489.

${ }^{16}$ Fedorov, A., Shiplyuk, A., Maslov, A., Burov, E., and Malmuth, N., "Stabilization of a hypersonic boundary layer using an ultrasonically absorptive coating," Journal of Fluid Mechanics, Vol. 479, 2003, pp. 99-124.

${ }^{17}$ Bountin, D., Chimitov, T., Maslov, A., Novikov, A., Egorov, I., Fedorov, A., and Utyuzhnikov, S., "Stabilization of a Hypersonic Boundary Layer Using a Wavy Surface," AIAA Journal, Vol. 51, No. 5, 2013, pp. 1203-1210. 
${ }^{18}$ Wagner, A., Kuhn, M., Martinez Schramm, J., and Hannemann, K., "Experiments on passive hypersonic boundary layer control using ultrasonically absorptive carbon-carbon material with random microstructure," Experiments in Fluids, Vol. 54, 2013, pp. 1606.

${ }^{19}$ Wagner, A., Hannemann, K., and Kuhn, M., "Ultrasonic absorption characteristics of porous carbon-carbon ceramics with random microstructure for passive hypersonic boundary layer transition control," Experiments in Fluids, Vol. 55, 2014, pp. 1750 .

${ }^{20}$ Wartemann, V., Wagner, A., Eggers, T., and Hannemann, K., "Passive hypersonic boundary layer control by means of ultrasonically absorptive carbon-carbon ceramics: Investigations of different boundary conditions, AIAA Paper 2015-3577," 20th AIAA International Space Planes and Hypersonic Systems and Technologies Conference, Glasgow, July 2015.

${ }^{21}$ Brès, G., Inkman, M., Colonius, T., and Fedorov, A., "Second-mode attenuation and cancellation by porous coatings in a high-speed boundary layer," Journal of Fluid Mechanics, Vol. 726, 2013, pp. 312-337.

${ }^{22}$ Zhao, R., Wen, C. Y., Long, T. H., Tian, X. D., Zhou, L., and Wu, Y., "Spatial Direct Numerical Simulation of the Hypersonic Boundary-Layer Stabilization Using Porous Coatings," AIAA Journal, Vol. 57, No. 11, 2019, pp. 5061-5065.

${ }^{23}$ Tritarelli, R. C., Lele, S. K., and Fedorov, A., "Stabilization of a hypersonic boundary layer using a felt-metal porous coating," Journal of Fluid Mechanics, Vol. 769, 2015, pp. 729-739.

${ }^{24}$ Zhao, R., Liu, T., Wen, C., Zhu, J., and Cheng, L., "Theoretical modeling and optimization of porous coating for hypersonic laminar flow control," AIAA Journal, Vol. 56, No. 8, 2018, pp. 2942-2946.

${ }^{25}$ Sousa, V., Patel, D., Chapelier, J.-B., Wagner, A., and Scalo, C., "Numerical investigation of second mode attenuation over carbon/carbon surfaces on a sharp slender cone, AIAA Paper 2018-0350," 2018 AIAA Aerospace Sciences Meeting, Kissimmee, January 2018.

${ }^{26}$ Sousa, V. C. B., Patel, D., Chapelier, J.-B., Wartemann, V., Wagner, A., and Scalo, C., "Numerical Investigation of Second-Mode Attenuation over Carbon/Carbon Porous Surfaces," Journal of Spacecraft and Rockets, Vol. 56, No. 2, 2019, pp. 319-332.

${ }^{27}$ Tian, X., Zhao, R., Long, T., and Wen, C. Y., "Reverse Design of Ultrasonic Absorptive Coating for the Stabilization of Mack Modes," AIAA Journal, Vol. 57, No. 6, 2019, pp. 2264-2269.

${ }^{28}$ Monteghetti, F., Matignon, D., Piot, E., and Pascal, L., "Design of broadband time-domain impedance boundary conditions using the oscillatory-diffusive representation of acoustical models," The Journal of the Acoustical Society of America, Vol. 140, No. 3, 2016, pp. 1663-1674.

${ }^{29}$ Vanharen, J., Puigt, G., Vasseur, X., Boussuge, J.-F., and Sagaut, P., "Revisiting the spectral analysis for high-order spectral discontinuous methods," Journal of Computational Physics, Vol. 337, 2017, pp. 379 - 402.

${ }^{30}$ Monteghetti, F., Matignon, D., and Piot, E., "Energy analysis and discretization of nonlinear impedance boundary conditions for the time-domain linearized Euler equations," Journal of Computational Physics, Vol. 375, 2018 , pp. 393 - 426.

${ }^{31}$ Fiévet, R., Deniau, H., and Piot, E., "Strong compact formalism for characteristic boundary conditions with discontinuous spectral methods," Journal of Computational Physics, in review.

${ }^{32}$ Poinsot, T. J. and Lele, S. K., "Boundary Conditions for Direct Simulations of Compressible Viscous Flows," Journal of Computational Physics, Vol. 101, 1992, pp. 104-129.

${ }^{33}$ Cardesa, J. I. and Airiau, C., "Automatic differentiation of a spectral difference code for sensitivity analysis," Bulletin of the American Physical Society, 2019.

${ }^{34}$ Cassagne, A., Boussuge, J.-F., and Puigt, G., "High-order Method for a New Generation of Large Eddy Simulation Solver," Technical report, PRACE, June 2015.

${ }^{35}$ Hamri, W., Evaluation of the JAGUAR solver with benchmark test cases, Toulouse, France, 2015.

${ }^{36}$ Lele, S. K., "Compact finite difference schemes with spectral-like resolution," Journal of Computational Physics, Vol. 103, No. 1, 1992, pp. $16-42$.

${ }^{37}$ Bogey, C. and Bailly, C., "A family of low dispersive and low dissipative explicit schemes for flow and noise computations," Journal of Computational Physics, Vol. 194, No. 1, 2004, pp. $194-214$.

${ }^{38} \mathrm{Kim}$, J. W. and Joo, D., "Generalized Characteristic Boundary Conditions for Computational Aeroacoustics, Part 2," AIAA Journal, Vol. 42, No. 1, 2004, pp. 47-55.

${ }^{39}$ Jones, M., Watson, W., and Parrott, T., "Benchmark Data for Evaluation of Aeroacoustic Propagation Codes with Grazing Flow, AIAA Paper 2005-2853," 11th AIAA/CEAS Aeroacoustics Conference, 2005.

${ }^{40}$ Douasbin, Q., Scalo, C., Selle, L., and Poinsot, T., "Delayed-time domain impedance boundary conditions (D-TDIBC)," Journal of Computational Physics, Vol. 371, 2018, pp. 50 - 66.

${ }^{41}$ Scherrer, D., Dessornes, O., Ferrier, M., Vincent-Randonnier, A., Sabel'nikov, V., and Moule, Y., "Research on Supersonic Combustion and Scramjet Combustors at ONERA," AerospaceLab Journal, , No. 11, 2016.

${ }^{42}$ Refloch, A., Courbet, B., Murrone, A., Villedieu, P., Laurent, C., Gilbank, P., Troyes, J., Tessé, L., Chaineray, G., Dargaud, J.-B., Quémerais, E., and Vuillot, F., "CEDRE Software," AerospaceLab Journal, , No. 2, 2011.

${ }^{43}$ Brazier, J.-P., Perraud, J., and Couzi, J., "Transition prediction on Reentry-F trajectory with PSE at chemical equilibrium," International Conference on Flight Vehicles, Aerothermodynamics and Re-entry Missions \& Engineering, Monopoli, oct 2019 .

${ }^{44}$ Toro, E. F., Spruce, M., and Speares, W., "Restoration of the contact surface in the HLL-Riemann solver," Shock Waves, Vol. 4, No. 1, Jul 1994, pp. 25-34.

${ }^{45}$ Esquieu, S., Benitez, E., Schneider, S. P., and Brazier, J.-P., "Flow and Stability Analysis of a Hypersonic Boundary Layer over an Axisymmetric Cone Cylinder Flare Configuration, AIAA Paper 2019-2115," AIAA Scitech Forum, 2019.

${ }^{46}$ Knisely, C. P. and Zhong, X., "Significant Supersonic Modes and the Wall Temperature Effect in Hypersonic Boundary Layers," AIAA Journal, Vol. 57, No. 4, 2019, pp. 1552-1566. 